\title{
A Gradient in Endogenous Rhythmicity and Oscillatory Drive Matches Recruitment Order in an Axial Motor Pool
}

\author{
Evdokia Menelaou and David L. McLean \\ Department of Neurobiology, Northwestern University, Evanston, Illinois 60208
}

\begin{abstract}
The rhythmic firing behavior of spinal motoneurons is a function of their electrical properties and synaptic inputs. However, the relative contribution of endogenous versus network-based rhythmogenic mechanisms to locomotion is unclear. To address this issue, we have recorded from identified motoneurons and compared their current-evoked firing patterns to network-driven ones in the larval zebrafish (Danio rerio). Zebrafish axial motoneurons are recruited topographically from the bottom of the spinal cord up. Here, we have explored differences in the morphology of axial motoneurons, their electrical properties, and their synaptic drive, to reveal how they match the topographic pattern of recruitment. More ventrally located "secondary" motoneurons generate bursts of action potentials in response to constant current steps, demonstrating a strong inherent rhythmogenesis. The membrane potential oscillations underlying bursting behavior occur in the normal frequency range of swimming. In contrast, more dorsal secondaries chatter in response to current, while the most dorsally distributed "primary" motoneurons all fire tonically. We find that systematic variations in excitability and endogenous rhythmicity are inversely related to the level of oscillatory synaptic drive within the entire axial motor pool. Specifically, bursting cells exhibit the least amount of drive, while tonic cells exhibit the most. Our data suggest that increases in swimming frequency are accomplished by the recruitment of axial motoneurons that progressively rely on instructive synaptic drive to shape their oscillatory activity appropriately. Thus, within the zebrafish spinal cord, there are differences in the relative contribution of endogenous versus networkbased rhythms to locomotion and these vary predictably according to order of recruitment.
\end{abstract}

\section{Introduction}

Vertebrate locomotion is executed by rhythmogenic networks in the spinal cord. The response of spinal motoneurons to rhythmic commands is a function of their electrical properties and the weight of their excitatory and inhibitory synaptic inputs (Grillner and Jessell, 2009; Harris-Warrick, 2010). While spinal neurons exhibit a range of conditional and intrinsic oscillatory properties (Schmidt et al., 1998; Sillar et al., 1998; Kiehn et al., 2000; Brownstone and Wilson, 2008; Ziskind-Conhaim et al., 2010; $\mathrm{Li}$, 2011), the relative contribution of endogenous versus network-driven rhythms to locomotion is poorly understood.

Physiological studies of spinal locomotor networks have been simplified by the relatively recent arrival of the zebrafish model system, due to the availability of transgenic lines and the ease with which neurons can be recorded from and subsequently identified in intact, behaving animals (Moreno and Ribera, 2010; Saint-

\footnotetext{
Received April 13, 2012; revised June 1, 2012; accepted June 18, 2012.

Author contributions: E.M. and D.L.M. designed research; E.M. and D.L.M. performed research; E.M. and D.L.M. analyzed data; E.M. and D.L.M. wrote the paper.

This work was supported by National Institute of Neurological Disorders and Stroke Grant R01-NS067299, The Esther A. and Joseph Klingenstein Fund, The Searle Scholars Program, and The Alfred P. Sloan Foundation. We are grateful to Joseph Fetcho, Jason MacLean, and members of the laboratory (Martha Bagnall, Sandeep Kishore, Wei-Chun Wang, and Bradley Lawrence) for comments on this manuscript and numerous discussions, to Stephen Ekker for providing us with the parg ${ }^{\text {mn2Et }}$ enhancer trap line, and to Matthew Chiarelli for all his work managing the fish facility.

The authors declare no competing financial interests.

Correspondence should be addressed to David L. McLean at the above address. E-mail: david-mclean@ northwestern.edu.

DOI:10.1523/JNEUROSCI.1809-12.2012

Copyright $\odot 2012$ the authors $\quad 0270-6474 / 12 / 3210925-15 \$ 15.00 / 0$
}

Amant, 2010; McLean and Fetcho, 2011; del Bene and Wyart, 2012). During swimming in larval zebrafish, smaller motoneurons nearest the ventral edge of spinal cord are recruited during the slowest movements, with progressively larger neurons recruited from the bottom up with increasing tail beat frequency (McLean et al., 2007). This topographic pattern of recruitment has links to development because the position of neurons along the dorsoventral axis is a reasonable reflection of their time of differentiation. Early-born "primary" motoneurons and excitatory premotor interneurons are located more dorsally, while younger "secondary" motoneurons and excitatory interneurons are added ventrally during development (Westerfield et al., 1986; Kimura et al., 2006; McLean and Fetcho, 2009; Satou et al., 2012).

Here, we have systematically recorded from axial motoneurons to see how their cellular properties and patterns of synaptic drive match the topographic functional organization of the axial motor pool. Our results reveal systematic relationships between the soma location, anatomy, physiology, and recruitment order of axial motoneurons. We find that, within the axial motor pool, neurons are organized dorsoventrally according to their endogenous rhythmicity. Moreover, this arrangement is inversely related to the level of oscillatory synaptic drive. Constant current injection reveals bursts of action potentials in the most ventrally distributed secondary motoneurons. Notably, the membrane potential oscillations underlying bursting behavior fall within the normal frequency range of locomotion. In contrast, more dorsally located secondaries exhibit a chattering response to current steps and lack intrinsic oscillations, while the most dorsally distributed primary motoneurons all fire tonically. Levels of oscilla- 
tory synaptic drive follow measures of excitability and the intrinsic firing patterns, with more excitable burst cells receiving the least amount of drive and less excitable tonic cells receiving the most. Our data suggest that increases in swimming frequency are accomplished by the recruitment of neurons that progressively rely on instructive synaptic drive to shape their oscillatory activity appropriately. Thus, within the zebrafish spinal cord, the relative contribution of endogenous versus network-based rhythms to locomotion varies predictably according to recruitment order.

\section{Materials and Methods}

Fish. Larval zebrafish were obtained from an in-house facility (Aquatic Habitats), containing wild-type and transgenic adults. Adult zebrafish and their offspring were maintained at $28.5^{\circ} \mathrm{C}$, but recordings were performed at room temperature $\left(\sim 22^{\circ} \mathrm{C}\right)$. Experiments were performed in zebrafish larvae between 4 and $5 \mathrm{~d}$ of age when they are free-swimming. At this stage, zebrafish have not yet sexually differentiated and are still nourished by their yolk. All procedures described below conform to NIH guidelines regarding animal experimentation and were approved by the Northwestern University Institutional Animal Care and Use Committee.

Electrophysiology and analysis. Electrophysiological recordings in zebrafish larvae were performed based on techniques described previously (Drapeau et al., 1999; Masino and Fetcho, 2005). Larvae were first immobilized by immersion in recording solution containing the neuromuscular blocker, $\alpha$-bungarotoxin $(0.1 \% \mathrm{w} / \mathrm{v}$; composition in $\mathrm{mmol} / \mathrm{L}$ : $134 \mathrm{NaCl}, 2.9 \mathrm{KCl}, 1.2 \mathrm{MgCl}_{2}, 2.1 \mathrm{CaCl}_{2}, 10 \mathrm{HEPES}, 10$ glucose, adjusted to $\mathrm{pH} 7.8$ with $\mathrm{NaOH} ; \alpha$-bungarotoxin purchased from Tocris Bioscience). After 5-10 min, immobilized larvae were transferred to a Sylgardlined glass-bottom dish and pinned down through the notochord using electrolytically sharpened tungsten pins. Next, the skin from just behind the ear to just past the anus was carefully removed using fine forceps. To provide access to spinal cells, muscle fibers covering the spinal cord were removed. A single muscle segment was carefully dissected away using a sharpened tungsten dissecting pin to ensure that the axon innervating muscle the next segment down was not severed. This was always confirmed after recordings.

After the dissection, the preparation was moved to the recording apparatus. Cells were targeted at $1000 \times$ magnification with a $40 \times / 1.0 \mathrm{nu}-$ merical aperture (NA) water-immersion objective on a compound microscope equipped with differential interference contrast (DIC) optics (AxioExaminer; Zeiss). Whole-cell recording electrodes were made using standard-wall $1 \mathrm{~mm}$ outer diameter borosilicate capillaries (A-M Systems), pulled to $0.5-1 \mu \mathrm{m}$ tip diameters (resistance, $15-20 \mathrm{M} \Omega$ ) using a micropipette puller (Flaming/Brown; Sutter Instrument). Suction electrodes for recording the activity of peripheral motor nerves were fashioned from these electrodes by cleanly breaking the tips to diameters of 20-50 $\mu \mathrm{m}$. A microforge (MF-830; Narishige) was used to simultaneously heat polish and bend the tip to compensate for the approach angle $\left(\sim 20^{\circ}\right)$, which improved the contact between the opening of the electrode and the muscle cleft.

Electrodes were lowered into place using motorized micromanipulators (MP-225; Sutter Instrument; or PatchStar; Scientifica). For whole-cell recordings, pipettes were backfilled with patch solution (composition in mmol/L: $125 \mathrm{~K}$-gluconate, $2.5 \mathrm{MgCl}_{2}, 10 \mathrm{EGTA}, 10 \mathrm{HEPES}, 4$ $\mathrm{Na}_{2} \mathrm{ATP}$, adjusted to $\mathrm{pH} 7.3$ with $\left.\mathrm{KOH}\right)$, and positive pressure $(\sim 20-40$ $\mathrm{mmHg}$ ) was maintained using a pneumatic transducer (DPM-1B; Fluke Biomedical) while the pipette was carefully advanced into the spinal cord. Once the spinal membrane was ruptured, the pipette tip was quickly brought into contact with the soma of the targeted cell and pressure was gradually reduced to atmosphere. Usually, at this point, a gigaohm seal was obtained, but if not a small amount of oral suction $(\sim 5-10 \mathrm{mmHg})$ was delivered to achieve the seal. When the pipette was cell-attached, a holding current was applied to maintain the cell at -65 $\mathrm{mV}$ and brief oral suction pulses were delivered to rupture the seal. Cells were targeted in the first or second cell layer. For recordings of peripheral motor nerve activity, the suction electrode was filled with recording solution and gently lowered onto the muscle cleft, usually just dorsal to the midpoint as this was likely to sample the largest number of axons. Typically, the capillary action of the electrode was enough to obtain a good seal; however, sometimes a small amount of suction $(<5 \mathrm{mmHg})$ was applied to improve the recording. To evoke faster swimming bouts, a tungsten bipolar electrode was lowered onto the tail skin using a manual micromanipulator (YOU-3; Narishige) and brief electrical stimuli (1-10 $\mathrm{V} ; 0.5-1 \mathrm{~ms}$ ) were delivered via an isolated stimulator (model 2100; A-M Systems; or DS2A-Mk.II; Digitimer). Swimming was also evoked by rapid changes in illumination with a flashlight.

Whole-cell recordings were acquired using a Multiclamp 700B amplifier, a Digidata series 1322A digitizer, and pClamp software (Molecular Devices). Standard corrections for bridge balance and electrode capacitance were applied in current-clamp mode. Electrical signals from spinal cells were filtered at $30 \mathrm{kHz}$ and digitized at $63 \mathrm{kHz}$ at a gain of 20 (feedback resistor, $500 \mathrm{M} \Omega$ ). Extracellular signals from the peripheral motor nerves were recorded at a gain of 1000 with low- and highfrequency cutoffs set at 100 and $4000 \mathrm{~Hz}$, respectively.

All electrophysiological data were analyzed off-line using DataView (Heitler, 2009). To determine the intrinsic firing patterns of cells, 500ms-long depolarizing current pulses were applied until spike threshold was reached (rheobase), and then levels were increased at regular intervals up to a maximum of $4 \times$ rheobase. Zebrafish larvae often swim spontaneously and this could give a false impression of intrinsic rhythmicity if bouts arrived coincident with current steps. We were careful to perform current injection when the larvae were not swimming and we could confirm the absence of swimming activity using the peripheral motor nerve recording, in addition to the whole-cell recording itself. Cells in which spontaneous activity was prevalent during current injection protocols were excluded.

Spike frequency measures represent the instantaneous frequency of the first two spikes for both current-evoked and synaptically evoked firing. Spike threshold was determined for the first spike at rheobase in current protocols, and for every spike during fictive swimming, by taking the double derivative of the spike waveform, which provides an objective measure of the fastest change in voltage. Measures of spike amplitude are from threshold values to the peak of the spike. For the spike afterhyperpolarization (AHP), we calculated the voltage deflection from the trough of the AHP to spike threshold. Spike half-widths represent the duration of the spike at one-half of the spike height. For bursting neurons, the frequency of bursts was measured using the interval between the first spike in each burst. Burst amplitude was measured from the threshold of the first spike in the burst to the trough following the last spike in the burst. To objectively pick out bursts, we used a thresholding/minimum interval function in DataView and modified it manually until it most accurately captured burst events, as confirmed by eye. The same value was then used throughout the current protocol. The error rate for burst detection increased with increasing current, and this represented a deterioration of the bursting behavior that matched a progressive difficulty in detecting bursts by eye. Values for bursting are only reported when they could be unambiguously identified, which were typically up to $1.3-1.5 \times$ rheobase. Input resistance was determined by averaging between 5 and 10, 500-ms-long hyperpolarizing pulses within a linear range of the current-voltage relationship (20-50 pA), as described previously (McLean et al., 2007).

To compare current-evoked behavior to synaptically evoked responses, we measured $10-15$ bouts of swimming per larvae to ensure sampling of as broad a range of swimming frequencies as possible. Reports of swimming frequency are the inverse interval of the onset of each cyclical motor burst. For membrane potential oscillation amplitudes, we measured the peak depolarization at on-cycle (which coincides with ispilateral motor bursts) and the peak hyperpolarization at midcycle (which coincides with contralateral motor bursts). If a neuron fired an action potential, we used the threshold value as the peak on-cycle depolarization. For suprathreshold on-cycle measurements, values indicate the depolarization from the resting membrane potential to spike threshold on a cycle-by-cycle basis.

Because the patch recording approach completely perfuses cells with dye-filled solution, it was easy to identify motoneurons after our recordings (see below, Imaging and analysis). Complete dialysis took on the 
order of 10-15 min. We were concerned about the potential effects of dialysis on electrical properties (Robinson and Cameron, 2000; Husch et al., 2011). To minimize this potential problem, we restricted our analysis to current steps delivered within the first minute of breaking into the cell. Immediately afterward ( $\sim 5 \mathrm{~min}$ in total), we would assess firing during fictive swimming activity. Unfortunately, it is impossible to rule out some contribution of dialysis to the firing patterns we report here. However, any effects should be fairly consistent from cell to cell since they were treated identically. To be considered healthy and included in the analysis, cells had to have resting membrane potentials below $-55 \mathrm{mV}$. This value represents a post hoc adjustment accounting for a liquid junction potential of $-16 \mathrm{mV}$ calculated previously using identical recording solutions (Higashijima et al., 2004). Values for resting membrane potential and spike threshold presented in Results have also been adjusted accordingly. No attempt was made to hold cells at a particular membrane potential, and those that depolarized $>5-10 \mathrm{mV}$ during the course of the first 5 min were excluded from the analysis.

Imaging and analysis. After recordings, the identity of the cell was confirmed by switching to an epifluorescent light source. Neurons would fluoresce because the patch solution also included a small amount of dye $(0.025 \%$ sulforhodamine-B acid chloride; Sigma-Aldrich). Images of cells were captured using a cooled CCD camera (Rolera-XR; QImaging) and a rhodamine filter set. To determine the muscle innervation territory, we collected a series of images at lower magnification (400×), gradually focusing down from the surface of the muscle to the spinal cord. To determine soma location and morphology, we collected a series of images at higher magnification $(1000 \times)$, gradually focusing down from the muscle to the far side of the spinal cord. In both cases, we captured fluorescent and DIC images. Soma position was based on an average of three measurements of the middle of the cell body normalized to an average of three measurements of the dorsal and ventral extent of the spinal cord (for dorsoventral measurements) or to the point at which the axon crossed the bottom edge of the spinal cord (for rostrocaudal measurements) using ImageJ software. To be included in the analysis, the borders of spinal cord had to be obvious and undamaged, and the cells had to be captured at perfectly right angles, as determined by completely overlapping Mauthner cell axons in the DIC image (these axons run along both sides of spinal cord). Cross-sectional area was also determined from three measures using ImageJ software (for soma size measurements). Reconstructions of neurons are based on a series of fluorescent images and were traced using Adobe Illustrator using $z$-compression stacks made in ImageJ. Unfortunately, since we manually focused down through the tissue to collect these images, we could not accurately quantify the depth at which axon collaterals terminated. Instead, we refer qualitatively to the known mediolateral organization of fast versus slow muscle fibers (for details, see Results).

To image the motoneuron enhancer trap line (Balciunas et al., 2004), we anesthetized parg $^{m n 2 E t}$ larvae in $0.02 \%$ MS-222 (ethyl 3-aminobenzoate methanesulfonic acid) in recording solution and then mounted them on their side in low-melting-point agarose within a glass-bottom dish. Once the agar had solidified, more anesthetic solution was placed in the dish to prevent agarose desiccation and movement artifacts. Imaging was performed using a confocal microscope (LSM710; Zeiss) with a $20 \times 1$ 1.0 NA water-immersion objective and a $488 \mathrm{~nm}$ laser to visualize green fluorescent protein (GFP). Series of images ( $z$-stacks) were collected at 1 $\mu \mathrm{m}$ intervals between body segments $10-13$. For illustration purposes, spinal $z$-stacks were reconstructed in three-dimensional volumes using Imaris 7.0 (Bitplane), and images were contrast inverted and cropped using Adobe Photoshop. Cell counts from parg ${ }^{m n 2 E t}$ larvae were performed in ImageJ. We were conservative in our approach and counted only the brightest GFP-labeled cells.

Statistical analysis. Before statistical analysis, all data were tested for normality to determine whether parametric versus nonparametric examinations were appropriate. Consequently, comparisons of the means between two groups were performed using a paired two-sample $t$ test, multiple comparisons were performed using a one-way ANOVA, followed by a post hoc Tukey-Kramer test, and correlations were determined using a Spearman rank $R$ correlation test. Degrees of freedom are reported parenthetically with the respective $t, F$, or $R$ values of these tests, according to convention. Statistical analysis was performed using StatPlus Professional (AnalystSoft) in conjunction with Microsoft Excel. All data are reported as means \pm SE, unless stated otherwise. Significance was set at $p<0.05$.

\section{Results}

To provide a comprehensive, unbiased picture of the axial motor pool, we systematically targeted motoneurons over the entire extent of the spinal segment. Recordings were performed in midbody segments 11-13 to minimize potential anatomical variability along the rostrocaudal length of spinal cord (Fig. $1 A$ ). Because motoneurons innervate the trailing muscle segment (Fig. 1B), careful dissection of the dorsal muscle provided access to somata

\section{A 4-5 day old larval zebrafish}

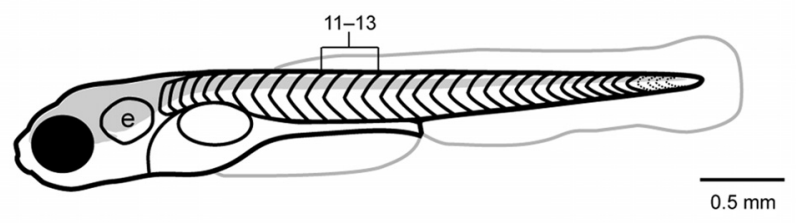

B parg $^{m n 2 E t}$ motoneurons

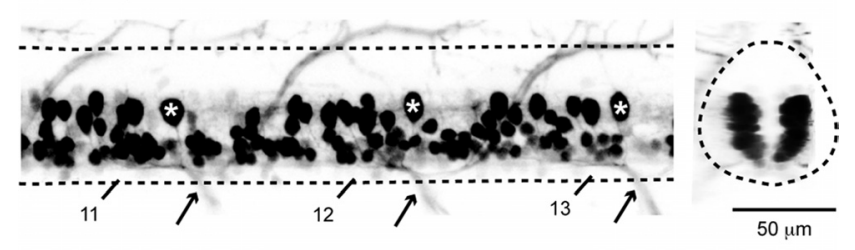

\section{Dissection}

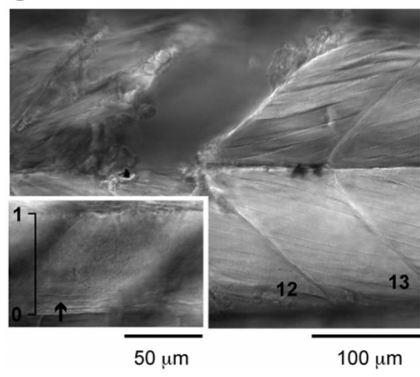

D

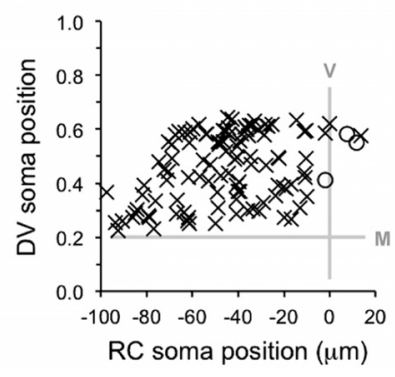

Figure 1. Systematic sampling of axial motoneurons throughout the spinal segment. $\boldsymbol{A}$, Schematic of a larval zebrafish at the developmental stage examined here ( $4-5 \mathrm{~d}$ of age). Larvae have just over 30 chevron-shaped muscle segments that begin just behind the ear (labeled " $\mathrm{e}$ "). The brain and spinal cord are shaded in gray. Motoneurons were targeted within the same region at midbody (segments 11-13). Here and elsewhere, rostral is to the left and dorsal is up. $\boldsymbol{B}$, A contrast inverted confocal image of a zebrafish larva from the parg ${ }^{\mathrm{mn} 2 \mathrm{Et}}$ enhancer trap line in which GFP is expressed in motoneurons. The image on the left illustrates the anatomical reference points used to calculate somata location, namely the top and bottom edges of spinal cord (dashed lines) and the ventral root exit point (arrows). The asterisks indicate likely caudal primary motoneurons ( $\mathrm{CaPs}$ ). The caudal edge of the muscle segments is numbered. For clarity, only the left side of the larva is illustrated (confocal $z$-stack depth, 43 $\mu \mathrm{m})$. On the right is an optical cross section from the same larva. Both the left and right pools of motoneurons are illustrated from the 12th segment. The dashed line indicates the outline of the spinal cord. C, Differential interference contrast image of the dissection for targeted whole-cell recordings. The inset image is at a different focal plane to illustrate access to the spinal cord for patch electrodes. The dorsal and ventral edges of spinal cord are normalized from 1 to 0 , respectively, for anatomical measurements. Muscle segments are numbered. In the inset image, the Mauthner cell axon is indicated by the black arrow. $\boldsymbol{D}$, The plot illustrates every motoneuron analyzed in this study ( $n=106$; marked with X's) with respect to rostrocaudal (RC) position, measured from the ventral root exit point (vertical gray line, V), and dorsoventral (DV) position, normalized to the top and bottom edges of spinal cord. Also illustrated here are cells whose identity could not be determined because their axons were severed during the dissection ( $n=$ 3; marked with 0 's). These cells were not included in the analysis. The dorsal edge of the Mauthner cell axon (horizontal gray line, $M$ ) is provided as an additional anatomical reference. 
while leaving the axon and muscle termination fields intact (Fig. $1 C$ ). Soma location of each neuron was plotted with respect to dorsoventral and rostrocaudal landmarks: the top and bottom edges of spinal cord and the exit point of the ventral root. We assessed the utility of these landmarks by examining the parg ${ }^{m n 2 E t}$ enhancer trap line, in which motoneurons selectively express green fluorescent protein (Balciunas et al., 2004). At these stages of development, the motor column is located in the ventral twothirds of the spinal cord and is only one to two cell bodies thick (Fig. $1 B$ ). The 106 neurons we recorded from wild-type larvae cover the entire territory where motoneurons are found (Fig. $1 B-D)$. In a few cases $(n=3)$, motoneurons targeted in the most rostral portion of the spinal segment had severed axons, due to the nature of the dissection, and were not included in further analysis (Fig. $1 D$, circles).

Earlier estimations of the number of axial motoneurons in zebrafish larvae suggest a lower limit of 20 per hemisegment (Myers, 1985). Cell counts in the parg ${ }^{m n 2 E t}$ larvae yielded numbers closer to 40 per hemisegment $(39.7 \pm 0.5 ; n=24$ hemisegments from 5 different larvae). Even using this higher estimate, our dataset represents a relatively comprehensive sampling of the axial motor pool. We next examined differences in morphology among these axial motoneurons, which would provide clues to their function.

\section{Stereotyped differences in muscle termination fields define four classes of primary motoneurons}

It is well established that zebrafish axial motoneurons can be divided into primary and secondary classes based on when they differentiate and innervate their target musculature (Myers et al., 1986). Within the primary class, there are between three to four cells per hemisegment that have been identified based on the relative rostrocaudal position of their somata and the stereotyped region of the axial musculature they innervate. Caudal primary (CaP) motoneurons innervate ventral musculature, middle primary (MiP) motoneurons innervate dorsal musculature, and rostral primary (RoP) motoneurons innervate the muscle in between. Primary motoneurons also develop in this order. In addition, there is a fourth class, called variable primary (VaP), which is observed less reliably and innervates muscle in between the RoP and MiP. This class appears to develop around the same time as the CaPs (Eisen et al., 1990).

Targeting large neurons in the dorsal part of the motor column consistently revealed four different types of primary motoneurons (Fig. 2A). The first type resembles the CaP, with somata located caudally in the muscle segment (Fig. $2 B$ ) and regions of arborization restricted to the ventral musculature (Fig. $2 C)$. The second type resembles the MiP, with somata locations just rostral to the CaP motoneurons (Fig. $2 B$ ) and a termination field restricted to the dorsal musculature (Fig. 2C). There were two types of motoneurons that fit the RoP description, in that they were the most rostral (Fig. 2B) and between them filled in the muscle territory left vacant by the CaPs and MiPs (Fig. 2C). One could be a RoP and the other a VaP, but given the variable presence of the latter, it was odd that no RoPs overlapped both the $\mathrm{CaP}$ and the MiP muscle fields (Fig. 2C). Since VaPs were identified based on strict anatomical and developmental criteria in more rostral regions of spinal cord, we have opted to name these motoneurons ventrally projecting RoPs or "vRoPs" and dorsally projecting RoPs or "dRoPs."

Primaries are known to be larger than secondaries (Myers et al., 1986), so to further characterize the CaPs, MiPs, and RoPs, we examined their cross-sectional area. From CaPs to dRoPs, there
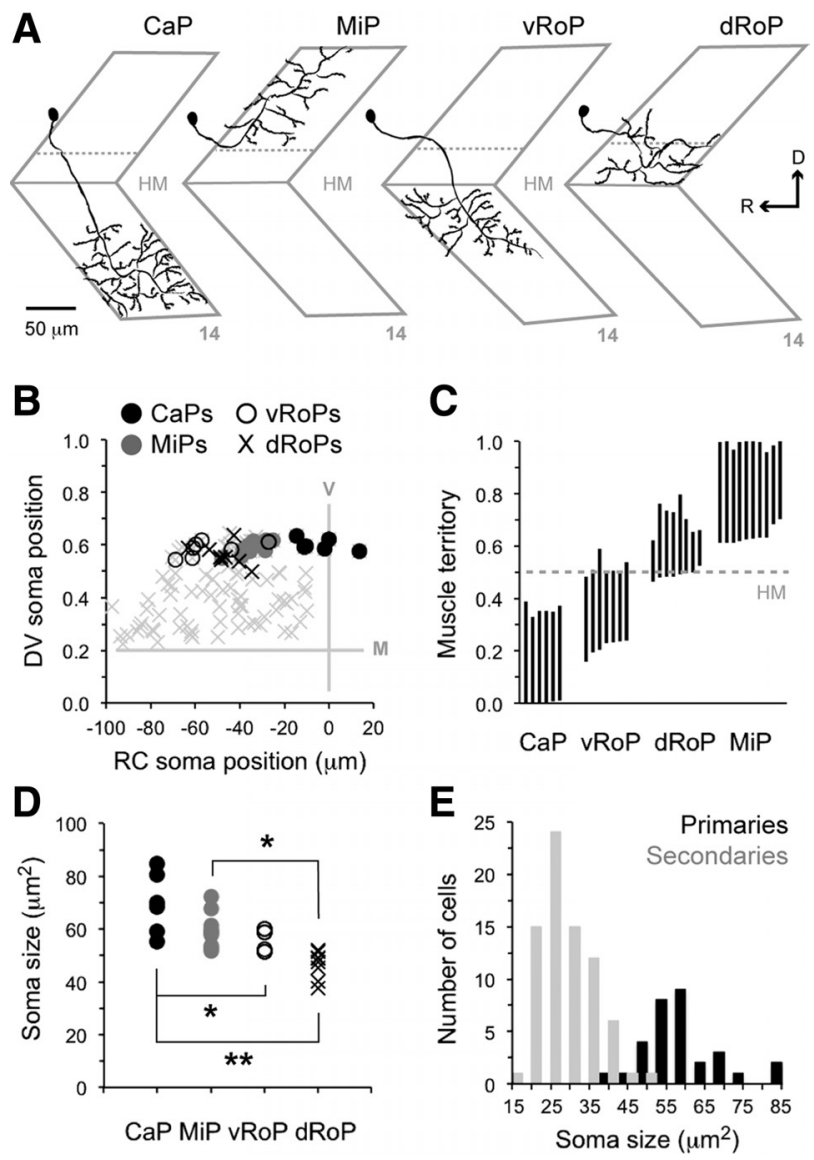

Figure 2. Four primary motoneurons tile the dorsoventral extent of the muscle segment. $\boldsymbol{A}$, Morphological reconstructions of primary motoneurons illustrate the somata, the main axon, and axon collaterals within the muscle. Shown here are representative reconstructions of a caudal primary (CaP), a middle primary (MiP), a rostral primary that innervates ventral musculature (vRoP), and a rostral primary that innervates dorsal musculature ( $\mathrm{dRoP})$. Collectively, the four classes cover the full dorsoventral extent of the muscle segment. The dashed gray line is the bottom edge of the spinal cord. The horizontal myoseptum is also indicated as a solid gray line in each reconstruction (labeled "HM"). Muscle segments are numbered in gray. Dorsal (D) is up and rostral (R) is to the left. $\boldsymbol{B}$, Somata locations of all primary motoneurons $(n=31)$, separated by anatomical class and overlaid on the total population (gray $X^{\prime}$ 's). Consistent with their nomenclature, CaPs are the most caudal, with the MiPs falling between the CaPs and the RoP classes. V, Ventral root exit point; M, dorsal edge of the Mauthner axon. C, Quantification of the dorsal-most and ventral-most extent of primary motoneuron axon collaterals, normalized to the top (1) and bottom ( 0 ) edges of the innervated muscle segment, confirms the stereotyped innervation patterns of each primary class. The horizontal myoseptum (HM) is included as a dashed gray line for reference. $\boldsymbol{D}$, Soma size distribution for each primary class illustrates a progressive decrease in cross-sectional area from the CaPs to the dRoPs. ${ }^{* *} p<0.001$ and ${ }^{*} p<$ 0.01 using a post hoc Tukey-Kramer test. $\boldsymbol{E}$, Histogram of the distribution of soma size for all 106 motoneurons, categorized anatomically as primary $(n=31)$ and secondary $(n=75)$. At midbody, primary motoneurons tend to be larger than secondary motoneurons, although there is a region of overlap.

was a systematic decrease in soma size (Fig. $2 D)$, which proved statistically significant following a one-way $\operatorname{ANOVA}\left(F_{(3,27)}=\right.$ $12.89 ; p<0.001 ; n=31)$. Post hoc analysis using a Tukey-Kramer test revealed significant differences between the CaPs and RoP classes, as well as the MiPs and the dRoPs (Fig. 2D). When the size distribution of all 106 motoneurons was plotted according to morphological criteria, primaries and secondaries fell into two distributions, with a clear region of overlap between 35 and 55 $\mu \mathrm{m}^{2}$ (Fig. 2E). Thus, soma size was not as definitive a measure of primaries as we anticipated, but rather formed a continuum. We will continue to refer to the $\mathrm{dRoP}$ and vRoP as primaries because 
of their complementary patterns of muscle coverage to CaPs and MiPs, although we concede they could be very early-born secondaries.

Among the primaries, collaterals would project off the main axon and ramify extensively throughout the deep musculature. In zebrafish larvae, slow skeletal muscle fibers are located superficially and fast skeletal muscle fibers are located more medially. The two fiber types are also easily distinguished based on their relative orientations along the longitudinal axis (van Raamsdonk et al., 1978, 1982; Buss and Drapeau, 2000; Luna et al., 2004). The extensive innervation of deep musculature is consistent with paired recordings demonstrating connections between primaries and fast muscle fibers in larvae (Wen and Brehm, 2005) and adult fish (Westerfield et al., 1986).

Axon collaterals were not just restricted to the muscle but could also be found within the spinal cord. Figure 3 illustrates examples from both primary and secondary motoneurons. The collateral typically projected off the main axon just before it exited the spinal cord via the ventral root, terminating in either the spinal neuropil or soma layer (Fig. $3 A, B$ ). Motoneu-
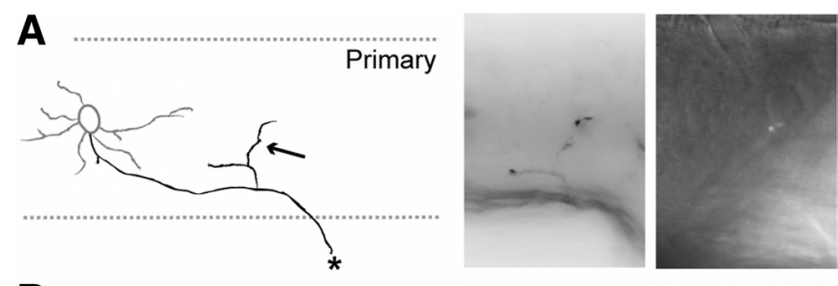

B
Secondary
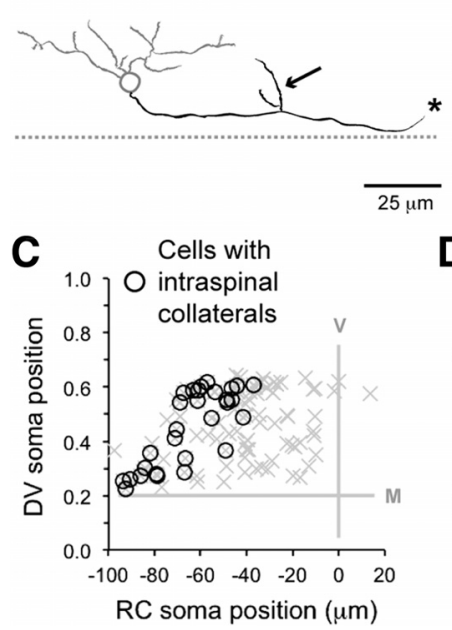

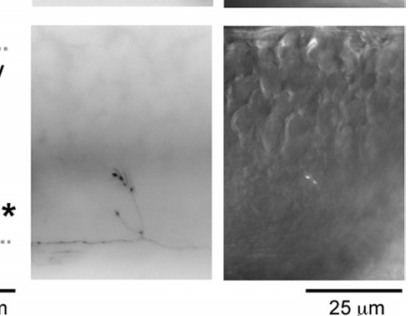

D

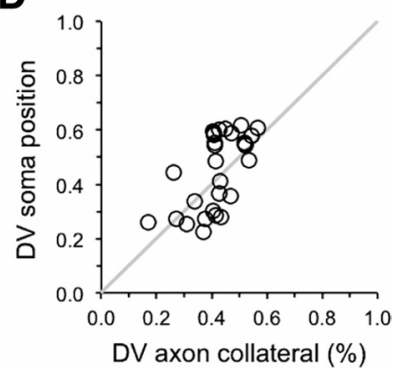

Figure 3. Primary and secondary motoneurons with intraspinal axon collaterals. $\boldsymbol{A}, \boldsymbol{B}, 0$ n the left are morphological reconstructions of a ventrally projecting $\operatorname{RoP}(\boldsymbol{A})$ and dorsally projecting secondary motoneuron $(\boldsymbol{B})$ with intraspinal axon collaterals (at arrows). The soma and dendrites are in gray, while the axon and intraspinal collaterals are in black. The dashed gray lines indicate the top and bottom edges of the spinal cord. The asterisks mark the continuation of the axons outside the plane of focus. In the middle are inverted epifluorescent images of the axon collaterals marked with arrows on the left. On the right, simultaneous differential interference contrast and epifluorescent images illustrate the location of the dorsal-most point of the axon collaterals, which can terminate in the spinal neuropil or soma layer. $\boldsymbol{C}$, Somata locations of all motoneurons with intraspinal collaterals $(n=29)$, which occupy more rostral regions of the spinal segment. The open circles are overlaid on the total population for reference (gray X's). V, Ventral root exit point; $M$, dorsal edge of the Mauthner axon. $D$, Measurements of the dorsoventral (DV) position of motoneuron somata versus the dorsal-most point of their respective intraspinal axon collaterals indicate a clear correlation between the two $\left(R_{(27)}=0.56 ; p<\right.$ $0.01 ; n=29)$. This suggests motoneurons can make contact with spinal cells at levels up to their own dorsoventral position. The solid gray line indicates unity. rons with axon collaterals tended to be located rostrally in the segment (Fig. 3C). The axon collateral from dorsally located cells consistently terminated dorsally, while the collateral from more ventral cells terminated more ventrally (Fig. $3 D$; $\left.R_{(27)}=0.56 ; p<0.01 ; n=29\right)$.

Primaries innervate fast muscle fibers and collectively cover the full dorsoventral extent of the muscle segment. Together with previous reports, these anatomical data confirm that primaries collectively activate muscles controlling faster, more powerful movements in the larval fish throughout the muscle segment. In addition, some motoneurons also have intraspinal collaterals, suggesting they could provide excitatory drive to spinal cells at and below their own dorsoventral position. These collaterals could serve to reinforce the topographic organization of recruitment observed in the larval spinal network (McLean et al., 2007). Next, we examined in more detail how secondary motoneurons may be distinguishable based on their soma locations and muscle termination fields.

\section{Secondary motoneurons have variable muscle targets}

Unlike primary motoneurons, far less is known about secondary motoneuron morphology with respect to muscle innervation territories (Menelaou and Svoboda, 2009). While it is known that distinct genes can identify secondaries with different muscle targets in transgenic lines (Higashijima et al., 2000; Balciunas et al., 2004; Uemura et al., 2005; Asakawa et al., 2012), it is not clear whether these differences are also related to soma location within the spinal cord. To be consistent with primary motoneuron anatomy, for purposes of comparison we have categorized secondaries based on their dorsoventral extent of muscle innervation (Fig. $4 A, B$ ).

The most dorsally located secondary motoneurons were distinct from identified primaries due to innervation fields in both the dorsal and ventral musculature (Fig. 4A, $C$, left). These cells also had more extensive branching off the main axon in deeper than more superficial locations, suggesting they preferentially activate fast over slow muscle. Of the presumed secondary motoneurons, these were the largest (up to $52 \mu \mathrm{m}^{2}$ ). Using the nomenclature introduced for the RoPs, we will refer to these motoneurons "dvS," for dorsoventrally projecting secondary, although we concede that their dorsal location and large size could indicate early differentiation. Moving ventrally (Fig. 4C, middle), the next type of motoneuron was one with a ventrally restricted arborization field (Fig. 4A, middle), which we call "vS," for ventrally projecting secondary. As with the dvS class, vS motoneurons also had axon collaterals that ramified deep in the muscle tissue. The final type with more extensive arborizations in fast skeletal muscle was one restricted to the dorsal musculature (Fig. $4 A$, right), which we call " $\mathrm{dS}$," for dorsally projecting secondary. These cells were typically found below the vS class and were concentrated in more rostral regions (Fig. 4C, middle). Collectively, the dvS, vS, and dS groups covered the full extent of the muscle segment with regions of overlap (Fig. 4D), as observed for primaries.

A second major group of secondaries was distinguishable based on a common anatomical feature: the presence of a major axon collateral that ran superficially along the intermyotomal boundary (Fig. 4B). We call this class "iS," for intermyotomal secondaries. There were at least two subgroups within the iS class. The first had extensive collaterals projecting off the main axon in both the deep and superficial musculature, which we designate as "iS-c," for intermyotomal secondaries with collaterals. The sec- 

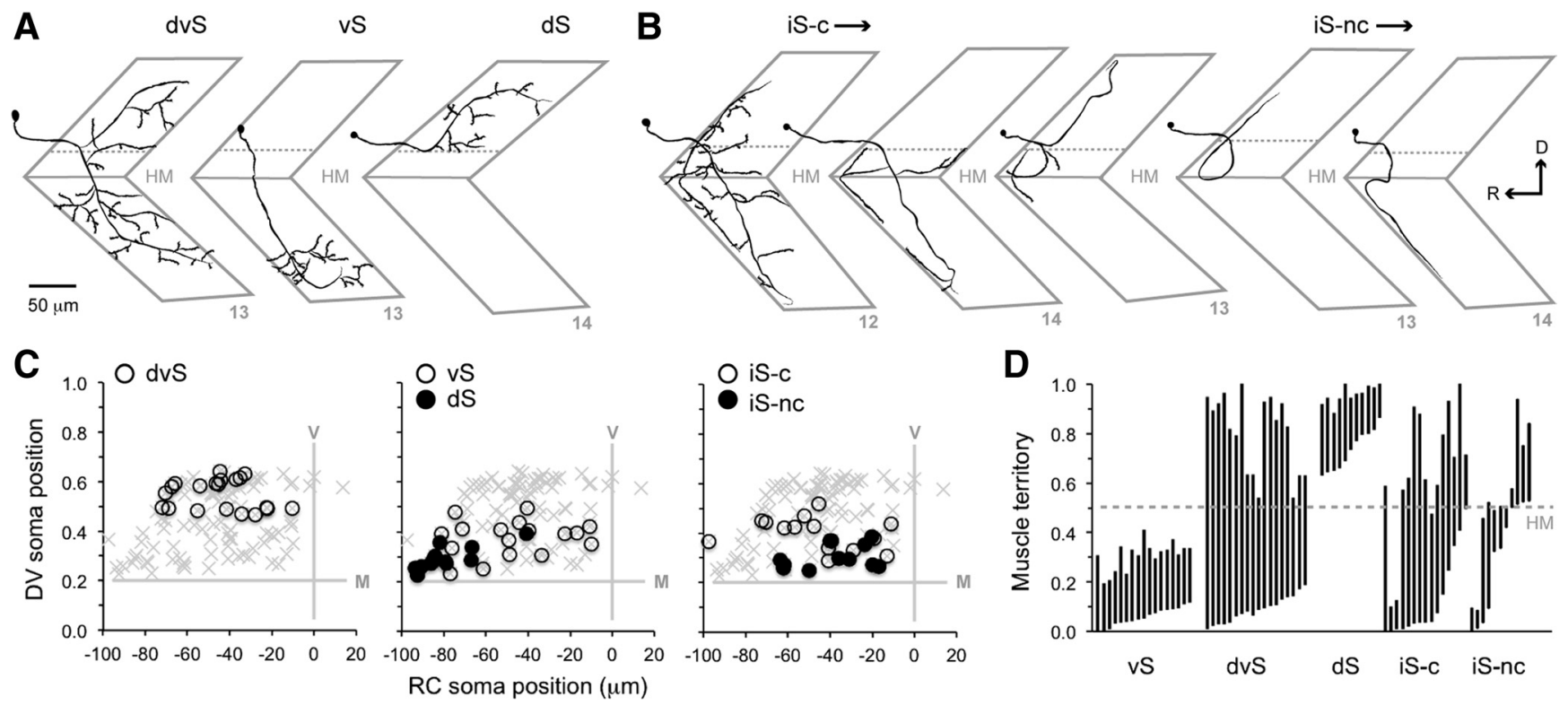

Figure 4. Arborization patterns can be used to categorize secondary motoneurons. $A$, Morphological reconstructions of secondary motoneurons illustrate the somata, the main axon, and axon collaterals within the muscle. Shown here are representative reconstructions of a dorsoventrally projecting secondary (dvS), a ventrally projecting secondary (vS), and a dorsally projecting secondary (dS). Collectively, these three classes cover the full dorsoventral extent of the muscle segment. The dashed gray line is the bottom edge of the spinal cord. The horizontal myoseptum (HM) is also indicated as a solid gray line. Muscle segments are numbered in gray. Dorsal (D) is up and rostral ( $R$ ) is to the left. $B$, Morphological reconstructions of secondary motoneurons with axons that run along the intermyotomal boundaries. Shown here are three intermyotomal secondaries with collaterals (iS-c) and two intermyotomal secondaries with no collaterals (iS-nc). C, Somata locations of all secondary motoneurons $(n=75)$. The dvS class is the most dorsally distributed one in the spinal segment, followed by vS and the dS classes. For the iS class, motoneurons with axon collaterals are distributed more dorsally than ones without them. For reference, data points are overlaid on the total population (gray X's). V, Ventral root exit point; M, dorsal edge of the Mauthner axon. $\boldsymbol{D}$, Quantification of the dorsal-most and ventral-most extent of secondary motoneuron axon branches, normalized to the top (1) and bottom (0) edges of the innervated muscle segment, confirm the overlapping muscle coverage provided by each class. For intermyotomal secondaries, the dorsal- and ventral-most points at which the axon reached the intermyotomal boundary were used for measurements. The HM is included as a dashed gray line for reference.

ond had only a single axon arbor, which terminated superficially along the intermyotomal boundary. We call this subgroup "iSnc," for intermyotomal secondary with no collaterals. The presence of an axon that runs superficially strongly suggests that one of the major muscle targets of this class is the slow skeletal muscle fibers.

Differences in the extent of muscle innervation between these iS subtypes were also associated with differences in their soma location: iS-c motoneurons were more dorsally located in the spinal cord than iS-nc ones (Fig. 4C, right). As with the primaries and the other secondaries, collectively the iS motoneurons tiled the full extent of the muscle segment (Fig. 4D).

Unlike primaries, there is no systematic rostrocaudal relationship for soma position according to region of muscle innervation among secondaries. Instead, different morphologies are organized along the dorsoventral axis. Among the secondaries are cells that innervate both fast and slow muscle fibers to varying degrees, as well as ones that appear to exclusively innervate the slow muscle fibers. Thus, axial motoneurons are organized according to their muscle targets and this arrangement is most clear along the dorsoventral axis. The graded, anatomical overlap likely ensures smooth transitions in muscle contractile strength during swimming. We have used relatively objective criteria to categorize motoneurons (e.g., crossing the horizontal myoseptum or the presence of intermyotomal axon); however, given the systematic variations in the dorsoventral extent and depth of muscle coverage, our anatomically defined classes more likely represent a functional continuum. We next investigated the responses to current injection within the axial motor pool.

\section{Motoneurons exhibit variable firing responses to current injection}

In Figure 5, we have provided examples of firing responses we typically observed in response to increasing steps of depolarizing current. To categorize firing classes, we focused on their patterns of firing during the entire $500 \mathrm{~ms}$ constant current step up to $2 \times$ rheobase values (Fig. $5 A-D$ ). Using this window of current injection, we could group motoneurons into three broad categories.

The first response we call "tonic," which was characterized by stable period of firing above $1.5 \times$ rheobase values (Fig. $5 A$ ). Tonic cells would often fire a single spike at rheobase ( $n=28$ of 31 cells), although in the remainder two or more spikes were observed ( $n=3$ of 31 cells). At levels of current above rheobase, there was a rapid accommodation of firing frequency, followed by steady-state firing that continued throughout the entire step (Fig. 5E). The later, stable period of high-frequency firing was also current dependent; higher amounts of current would lead to higher steady-state frequencies of spiking (Fig. $5 E$ ). At levels of current exceeding $2.5 \times$ rheobase, tonic neurons would begin to show accommodation and could cease firing before the end of the current step (data not shown).

Plotting the instantaneous firing rate of the first two spikes as a function of current injection revealed that tonic cells sharply increased their firing rate in response to increased current and began to level off above $2 \times$ rheobase (Fig. $5 H$ ). The response showed little variability from cell to cell when normalized to rheobase. A comparison of the spike rate at the lowest and highest level of current tested revealed a statistically significant increase in firing frequency in response to current $\left(t_{(30)}=35.16\right.$; $p<$ $0.001)$, from $94.6 \pm 6.9$ to $706.4 \pm 16.3 \mathrm{~Hz}(n=31)$. 

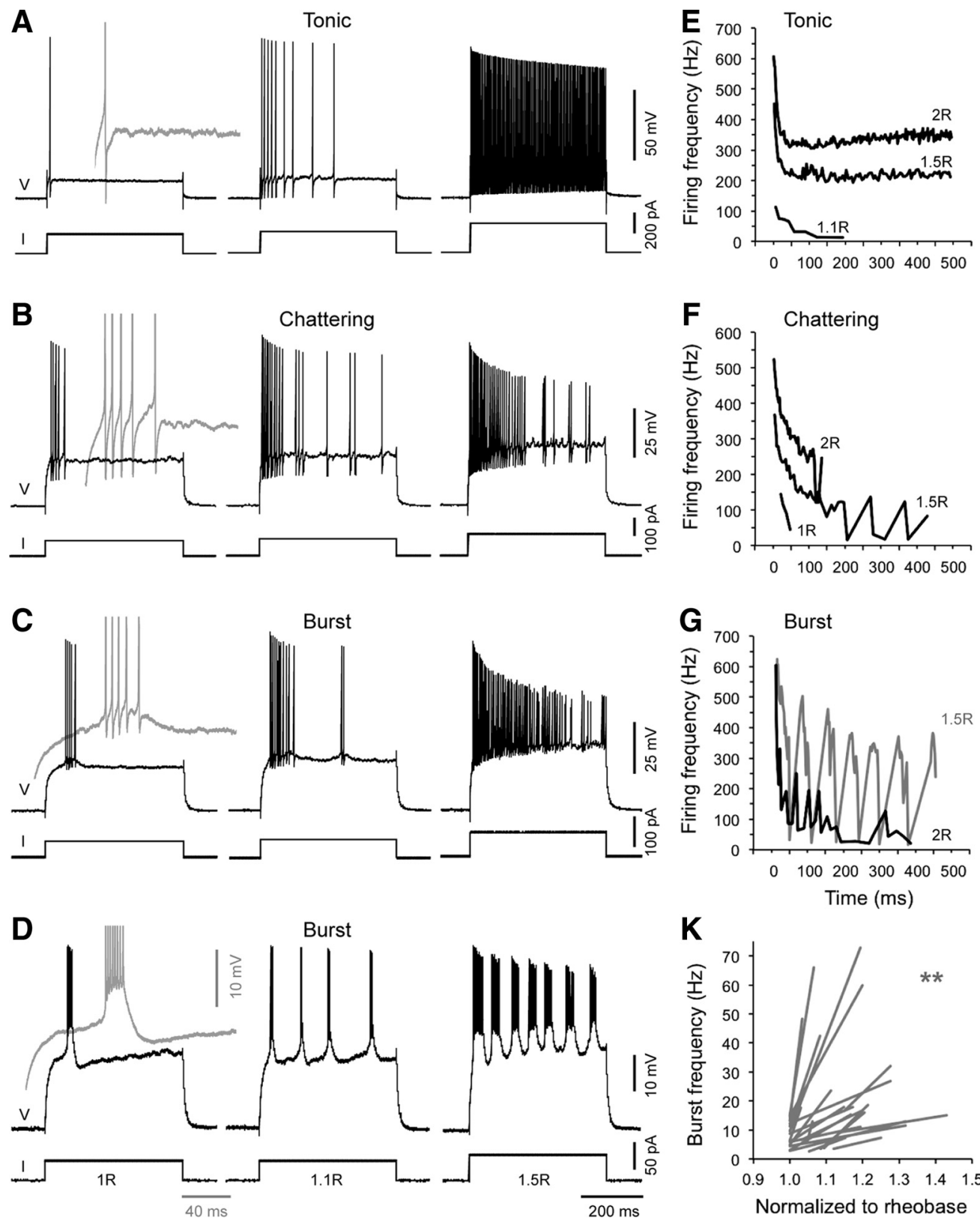
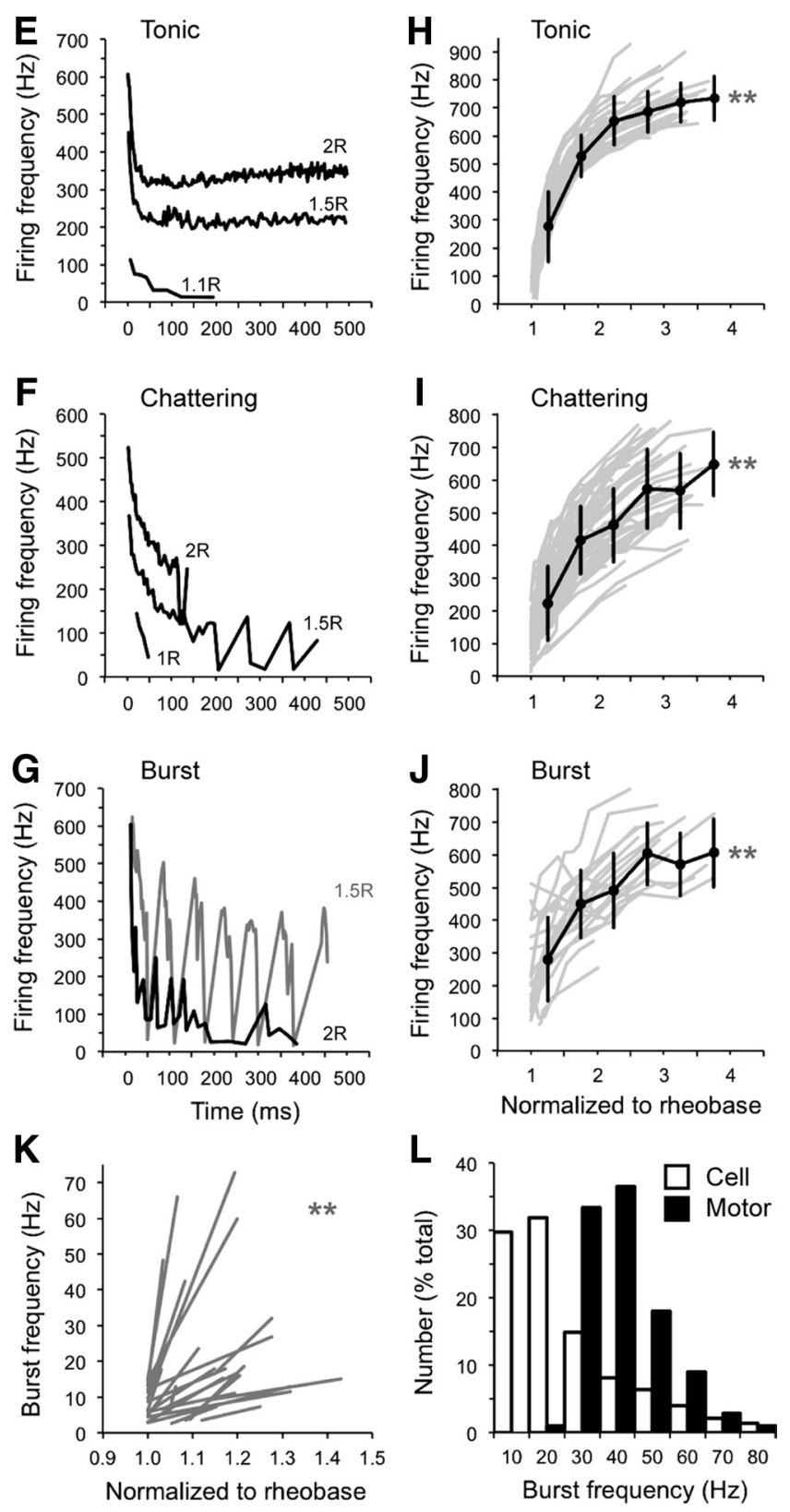

Figure 5. Three common responses of motoneurons to current injection. $\boldsymbol{A}-\boldsymbol{D}$, Examples of a tonic response in a CaP motoneuron $(\boldsymbol{A})$, a chattering response in a dvS motoneuron ( $\boldsymbol{B})$, and burst responses in avS motoneuron $(\boldsymbol{C})$ and dS motoneuron $(\boldsymbol{D})$. Each response is shown at 1,1.1, and $1.5 \times$ rheobase $(\mathrm{R})$ from left to right. There is a progressive increase in the complexity of the firing responseto current steps from tonic to burst cells. Rheobase for the CaP was $300 \mathrm{pA}$, for the dvS was $105 \mathrm{pA}$, for the vS was $65 \mathrm{pA}$, and for the dS was $35 \mathrm{pA}$. Voltage ( $V$ ) and current ( $/$ ) calibration bars are to the right, while time is presented below in $\boldsymbol{D}$. The inset in gray on the left is the response of each class at rheobase on a faster timescale to more clearly illustrate the slower depolarization that characterizes the endogenous membrane potential oscillations of burst responses. Traces are all at the same calibration as indicated in $\boldsymbol{D}$. Note that all voltage records include small artifacts aligned with the onset and offset of the current step, due to capacitance compensation and bridge balance correction. $\boldsymbol{E}-\boldsymbol{G}$, The firing frequency over the entire 500 ms current step for the tonic $(\boldsymbol{E})$, chattering $(\boldsymbol{F})$, and bursting $(\boldsymbol{G})$ cells in $\boldsymbol{A}, \boldsymbol{B}$, and $\boldsymbol{D}$ is plotted, with responses labeled as a function of rheobase (e.g., $2 R=2 \times$ rheobase). Tonic motoneurons can fire at stable levels throughout suprathreshold current steps, while chattering and burst cells tend to rapidly accommodate at higher levels of current injection. For clarity, we have only presented the burst response in $\mathbf{G}$ at 1.5 and $2 \times$ rheobase. $\boldsymbol{H}-\boldsymbol{J}$, The instantaneous firing frequency of the first two spikes in response to increasing levels of current is plotted for tonic $(\boldsymbol{H} ; n=31)$, chattering $(\boldsymbol{I} ; n=53)$, and bursting motoneurons $(\boldsymbol{J} ; n=22)$. All firing classes showed an increase in firing frequency in response to increased current steps. Each response is normalized to their respective rheobases. The gray lines indicate the individual motoneuron responses, while the black lines with SDs are an average response of all cells binned at 0.5 rheobase intervals. The double gray asterisks indicate significant differences using a pairwise comparison of firing frequency at the lowest and highest value of current tested on a cell-by-cell basis (see Results for details). $\boldsymbol{K}$, For burst neurons, the instantaneous frequency of the first two bursts is plotted at the lowest and highest level of current where bursting was observed ( $n=22$ ). Each response is normalized to rheobase. The double gray asterisks indicate a significant increase in burst frequency in response to increased current using a pairwise comparison (see Results for details). $L$, The instantaneous frequency of the every cellular burst over the entire 500 ms current step ( 1065 bursts from 22 cells) compared with the frequency of motor bursts recorded from peripheral motor nerves during fictive swimming ( $n=2230$ cycles from 7 different larvae). The cellular bursting data represent multiple current steps over the narrow range where bursting was most easily detected (from 1 to $1.5 \times$ rheobase). Data are normalized to the total number of observations in 10 $\mathrm{Hz}$ bins (\% total). The intrinsic bursting behavior occurs at frequencies that overlap with motor bursts generated during fictive swimming.

The second response we call "chattering" (Fig. $5 B$ ) because in contrast to the stable firing of tonic cells between 1.5 and $2 \times$ rheobase, chattering cells fired intermittent spikes whose frequency accommodated more rapidly. The more sensitive accom- modation of spike frequency is what helped us distinguish the chattering pattern from the tonic one (Fig. $5 F$ ). Chattering motoneurons would fire a train of action potentials around rheobase that rapidly accommodated, followed by highly variable rates of 
firing beyond rheobase, whose frequencies never exceeded those observed at the onset of the current step (Fig. $5 F$ ). Chattering neurons would more often fire two or more spikes at rheobase ( $n=37$ of 53 cells), although in the remainder a single spike was observed ( $n=16$ of 53 cells). In general, chattering neurons did not respond as robustly to suprathreshold current injection and would often terminate spiking before the end of current step.

Despite the relatively high spike rate at rheobase, chattering cells still exhibited a near-linear increase in instantaneous firing frequency in response to current. Chattering cells could reach spike frequencies observed in tonic cells; however, there was a great amount of variability in the gain (slope) of the response and the peak frequencies, when compared with tonic ones (Fig. 5I). A comparison of the instantaneous firing frequency at the lowest and highest current step revealed a significant increase in the firing rate $\left(t_{(52)}=22.76 ; p<0.001\right)$, from $117.7 \pm 7.6$ to $555.2 \pm$ $18.7 \mathrm{~Hz}(n=53)$. The tonic and chattering responses are consistent with firing patterns described previously for larval zebrafish motoneurons (Buss et al., 2003).

The final response we call "burst," which was distinguished from the chattering response based on slower membrane potential oscillations underlying faster trains of spikes (Fig. $5 C, D$ ). We defined burst cells according to the presence of these clear membrane potential oscillations, in which the slower component comprised at least $10 \%$ of the total amplitude of the spike train (mean, $16.3 \pm 1.2 \%$; range, $10.3-28.2 \% ; n=22$ ). In contrast, spikes in tonic and chattering cells were followed by relatively stable membrane potentials (Fig. 5A-D, left inset). The slow depolarization increased the chances that burst cells would fire two or more action potentials at rheobase ( $n=21$ of 22 cells, with only one cell firing a single spike) and also led to a longer delay from current onset to the first spike, when compared with tonic and chattering cells (Fig. $5 A-D$, left). After an initial high-frequency spike burst, subsequent bursts would not exceed the rates observed at the onset of the current step (Fig. 5G). Above a certain level of current injection, however, the oscillatory bursting firing pattern deteriorated into one that was indistinguishable from the suprathreshold response in chattering cells; namely intermittent firing and rapid accommodation, where slower oscillations were no longer obvious (Fig. $5 C, G$ ). This typically occurred at levels above $1.3-1.5 \times$ rheobase. In response to increasing steps of current, burst neurons increased their firing rate in a nearlinear fashion, albeit at a variety of different gains (Fig. $5 \mathrm{~J}$ ). Again, by comparing the instantaneous spike rate at the lowest and highest current step, burst neurons significantly increased their firing rate $\left(t_{(21)}=7.74 ; p<0.001\right)$, from $232.8 \pm 27.5$ to $570.8 \pm 31.6 \mathrm{~Hz}(n=$ 22 ). While these bursting responses have not been described previously in larvae, a similar response to depolarizing steps of current has been observed in older zebrafish (Gabriel et al., 2011).

Within bursting neurons, we observed variability in the frequency of membrane oscillations from cell to cell (Fig. 5C,D), but in all cells the burst frequency demonstrated a clear sensitivity to current, increasing its rate in response to increased current (Fig. $5 D, K)$. A comparison of the burst rate at the lowest and highest level of current tested (up to $1.5 \times$ rheobase) revealed a significant increase $\left(t_{(21)}=5.24 ; p<0.001\right)$, from $7.7 \pm 0.9$ to $26.0 \pm 4.1 \mathrm{~Hz}$ $(n=22)$. The current dependence of the bursting response suggests that it is an endogenous property of the cell and can also be driven to frequencies observed during larval swimming (Buss and Drapeau, 2001; Müller and van Leeuwen, 2004). To more clearly illustrate this point, we compared the frequency of current-evoked bursts to the frequency of bursts of action potentials monitored from motoneuron axons during "fictive" swim- ming (Fig. 5L). Extracellular recordings of axonal motor bursts in $\alpha$-bungarotoxin-immobilized larvae are a reliable indicator of activity that would drive real swimming (Masino and Fetcho, 2005), so we will refer to motor nerve burst frequency as swim frequency. While the majority of the cellular bursting behavior occurred at or below the lowest frequencies observed during fictive swimming (15-20 Hz), intrinsic bursting was also observed at more functionally relevant frequencies $(20-80 \mathrm{~Hz})$. The fact that cellular bursting behavior can be driven to frequencies observed during fictive swimming is consistent with the idea that intrinsic responses in these cells could contribute to the final motor output.

In response to depolarizing current steps, the firing responses of axial motoneurons can be divided into three broad categories. There are progressive differences in the number of spikes at rheobase, the rhythmic behavior at suprathreshold levels, and the variability in the gain of firing from tonic to burst cells. The clearest difference in firing behavior was between the tonic and the burst categories, with chattering cells appearing to represent a transitional state. Among the most rhythmic responses (burst cells, defined based on the presence of endogenous membrane potential oscillations), the frequency range of cellular bursts overlaps with the frequencies of motor bursts observed during fictive swimming. We next investigated whether these firing responses reflected systematic differences in electrical properties and how they related to motoneuron anatomy.

\section{Firing patterns reflect a gradient of electrical and anatomical features}

We created three categories to emphasize the variability in firing behavior within the axial motor pool. However, these categories are not based on quantitative data, but rather a qualitative assessment of motoneuron responses at different levels of current injection. Several features of the initial action potential waveform at rheobase varied according to firing type (Fig. 6A), so we next used more objective measures of electrical properties to see whether there was any quantifiable justification for discrete classes.

Two properties that appeared to covary between firing classes were spike AHP and spike amplitude (Fig. 6A). When we plotted spike height against AHP, we found a significant positive correlation between the two properties $\left(R_{(104)}=0.91 ; p<0.001\right.$; $n=106$ ). However, instead of dividing into discrete groups according to firing type, there was a considerable overlap in these electrical properties (Fig. 6B). We next plotted spike half-width against rheobase, to see whether these properties would distinguish firing classes. Again, the relationship was significantly correlated $\left(R_{(104)}=-0.90 ; p<0.001 ; n=106\right)$, but no clear separation was observed between the classes (Fig. 6C). We then compared input resistance against soma cross-sectional area, to see whether this would reveal distinct classes (Fig. 6D). As you would predict, soma size was negatively correlated with input resistance $\left(R_{(104)}=-0.88\right.$; $p<0.001 ; n=106)$, but there was an overlap in values that was more consistent with a continuum rather than discrete classes. Thus, tonic cells were the biggest neurons, had the largest AHPs, the largest spikes, the shortest half-widths, the largest rheobases, and the lowest input resistances; burst cells populated the opposite end of the spectrum; and chattering cells fell in between. These observations are consistent with the gradual increase in the complexity of firing and the endogenous rhythmicity of the currentevoked response from tonic to burst cells (Fig. $5 A-D$, middle). They also suggest there are systematic differences in the relative density of 


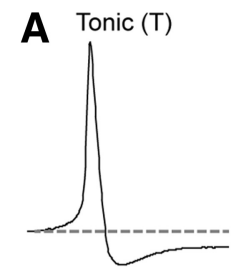

B
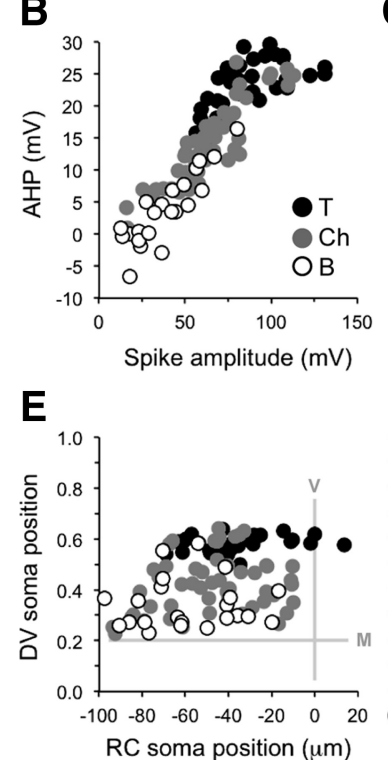

Chattering (Ch)

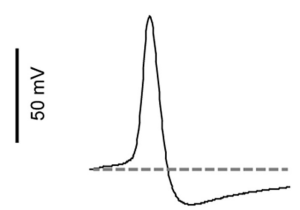

C

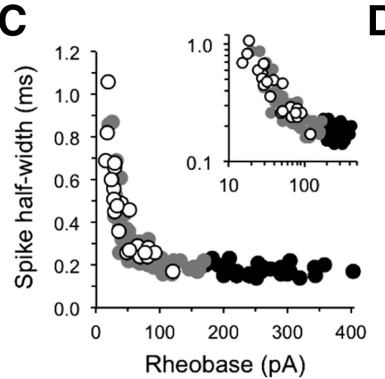

Burst $(\mathrm{B}) \longrightarrow$
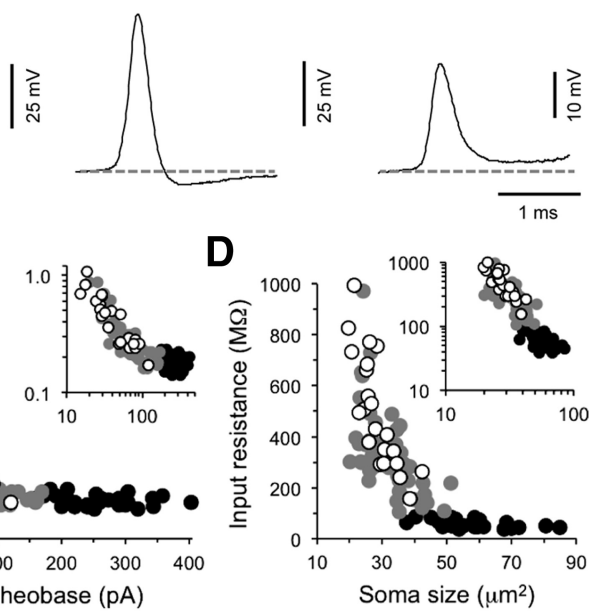

$\mathbf{F}$

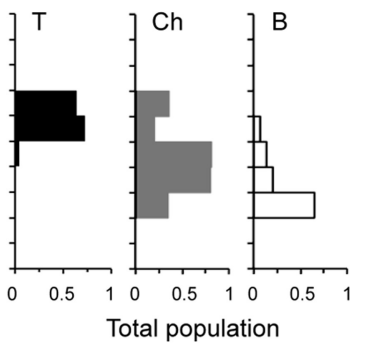

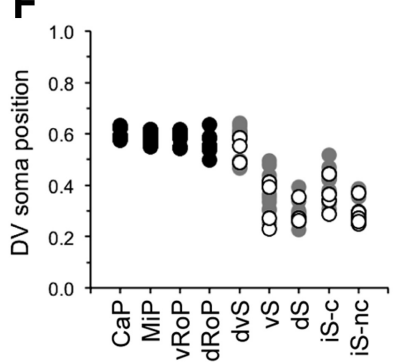

Figure 6. Current-evoked firing responses exhibit graded, overlapping measures of intrinsic excitability related to their morphologies. $A$, The first spike at rheobase for the tonic, chattering, and burst cells presented in Figure $5 A-D$. Note the progressive differences in spike amplitude, half-width, and AHP observed between the classes. A gray dashed line is provided for reference. $\boldsymbol{B}$, Spike AHP versus spike amplitude for each firing response. There is a clear correlation between the two properties when you examine the whole population $\left(R_{(104)}=0.91 ; p<0.001 ; n=106\right)$. There is also significant overlap between the grouped firing responses, which argues more for a continuum and less for discrete classes. The correlation is maintained when only the secondary motoneurons are examined $\left(R_{(73)}=0.91 ; p<0.001 ; n=75\right)$. C, Spike half-width versus rheobase for each firing response. There is a clear correlation between the two properties when you examine the entire population $\left(R_{(104)}=-0.90 ; p<0.001 ; n=106\right)$. The inset is the same plot on a logarithmic scale to make this point clearer (both $X$-and $Y$-axes). The distribution of values between firing responses is consistent with a continuum. The correlation is maintained when only the secondary motoneurons are examined $\left(R_{(73)}=-0.90 ; p<0.001 ; n=75\right)$. D, Input resistance versus soma size for each firing response. There is a clear correlation between the two properties when you examine the entire population $\left(R_{(104)}=-0.88 ; p<0.001 ; n=106\right)$. The inset is the same plot on a logarithmic scale to make this point clearer (both $X$ - and $Y$-axes). Again, a continuous gradient between the firing responses is evident. The correlation is maintained when only the secondary motoneurons are examined $\left(R_{(73)}=-0.73 ; p<\right.$ $0.001 ; n=75)$. $E$, On the left, quantification of the dorsoventral (DV) and rostrocaudal (RC) distribution of the somata of different firing responses. Tonic cells are more dorsally located, burst cells are more ventrally located, and chattering cells overlap the two classes. On the right, this relationship is more obvious when you plot firing class as a proportion of the total population at different dorsoventral locations (normalized from 1 to 0 ). V, Ventral root exit point; $M$, dorsal edge of the Mauthner axon. $F$, Dorsoventral distribution of different anatomical classes of motoneurons related to their firing responses. $\operatorname{CaP}(n=6), \operatorname{MiP}(n=10), \operatorname{vRoP}(n=$ 7), dRoP $(n=8), \mathrm{dvS}(n=20), \mathrm{vS}(n=18), \mathrm{dS}(n=11)$, iS-c $(n=15)$, and iS-nc $(n=11)$. Tonic cells are exclusively primary, while the chattering and burst cells are found among all anatomical classes of secondary motoneurons.

ion channels governing both active and passive membrane properties with the axial motor pool, which ultimately contribute to the progressive differences in firing patterns.

To see how the differences in electrical properties relate to motoneuron anatomy, the firing response of a cell was plotted according to its respective dorsoventral and rostrocaudal position (Fig. 6E, left). Tonic cells were distributed more dorsally, burst cells distributed more ventrally, and chattering cells overlapped the two (Fig. 6E, right). All classes were found along the full rostrocaudal extent of the spinal segment. The dorsoventral organization of the firing responses matches differences in the excitability and size for these motoneurons (Fig. 6D), as expected from previous work (McLean et al., 2007). Again, the overlap in dorsoventral position is consistent with the gradation in electrical features observed between the firing types.

In addition to the soma location, we determined the distribution of firing responses among our anatomical designations (Fig. $6 F$ ). When we matched firing responses to the different primary and secondary motoneuron classes, an explanation for the dorsal bias of tonic cells became obvious, since this was exclusively observed in primary motoneurons (Fig. $6 F$ ). Among the secondary classes, we found both chattering and burst cells. However, within each class, there was a tendency for the bursting response to populate the more ventral subset of cells (Fig. 6F). Between the secondary classes, the highest proportion of burst responses was found among the is cells (iS-nc, 73\%, $n=11$; iS-c, $27 \%, n=15)$, followed by the dS $(27 \% ; n=11)$, vS $(22 \% ; n=18)$, and then dvS cells $(15 \% ; n=20)$. This systematic shift in the relative occurrence of bursting versus chattering cells among anatomical classes is consistent with the differences in their dorsoventral location and explains the graded distribution we observe for the firing classes (Fig. $6 E$ ).

Following these anatomical observations, one potential concern was that the correlations we observed for electrical properties and size were due to the division of motoneurons into primary and secondary classes. To rule this out, we performed separate analyses for secondaries alone. All of the statistically significant observations were maintained when we excluded the primary population (for statistical data, see Fig. 6 legend). Thus, with the exception of primary motoneurons, there were no clear anatomical features that defined a particular firing class. Instead, firing responses followed the anatomical distribution along the dorsoventral axis, which was apparent even within the secondary motoneuron population.

The firing patterns and measures of neuronal excitability match the dorsoventral topography we have described here for anatomy and previously for recruitment (McLean et al., 2007). While primary motoneurons all fire tonically, secondary motoneurons exhibit a larger degree of heterogeneity in their firing properties. The increase in the proportion of endogenously bursting cells as you move ventrally also matches the greater role of more ventral motoneurons in activating slower muscle fibers. Although we will continue to use the firing terminology for purposes of comparison with network-driven activity, our data actually support a functional continuum. Our next goal was to see the extent to which differences in rhythmic drive matched the gradations in firing patterns, excitability, and morphology. 
Systematic differences in rhythmic drive and recruitment order match the electrical properties of motoneurons

To reveal differences in the rhythmic drive and recruitment of axial motoneurons during swimming, the voltage changes responsible for their activation were examined in current-clamp mode. Larval zebrafish will often swim spontaneously at lower frequencies, examples of which are shown on the left in Figure $7 A-D$. On top are the voltage records from the patched motoneuron, while below are the extracellular recordings of peripheral motor nerve activity that represents fictive swimming. On a faster time base, the rhythmic voltage oscillations that coincide with rhythmic bursts of swimming are illustrated at both lower (Fig. $7 A-D$, middle) and higher (Fig. $7 A-D$, right) swimming frequencies. Records include oscillations that were suprathreshold and caused the motoneurons to fire action potentials.

We first asked how spike frequency might vary as a function of swimming frequency among the different firing classes. As swimming frequency increases, motoneurons are less likely to fire two or more spikes (McLean et al., 2008), which makes spike frequency measures over the full range of frequencies difficult (Fig. $7 B, C$, right). However, in 89 of the 106 cells, we observed multiple spikes at fictive swimming frequencies up to $70 \mathrm{~Hz}$ and in all classes there was a consistent increase in spike frequency related to increases in swimming frequency (Fig. $7 E-G$ ). Given the considerable variability in spike frequency from cell to cell, pairwise comparisons were performed to determine whether this represented a significant difference. In all classes, spike frequency increased significantly (for statistical data, see Fig. 7 legend). This increase in spike frequency during increases in swimming frequency matches the response of these same neurons to increased levels of current and supports the idea that higher frequencies of swimming are accompanied by increases in synaptic drive.

During this more systematic examination of axial motoneurons, it was apparent that some motoneurons did not continue to fire at higher swimming frequencies (Fig. 7D). This behavior did not match firing class, but rather was related to morphology. In all iS-nc motoneurons $(n=11)$, there was a progressive drop in the reliability of firing at high swim frequencies, which was not observed in the functionally related iS-c motoneurons (Fig. 7H). At higher swim frequencies, these cells failed to reach threshold (Fig. $7 D$, right) and instead received a barrage of synaptic activity similar to that observed in premotor excitatory interneurons when they are silenced by inhibition (McLean et al., 2008). This stands in contrast to previous reports that all motoneurons are progressively recruited into the active pool (McLean et al., 2008). However, when the reliability of firing was instead plotted as a function of firing class, all motoneurons showed a progressive increase in recruitment despite the loss of iS-nc cells (Fig. 7I). Burst cells fired most reliably at lower swimming frequencies, followed by chattering cells and then tonic ones. So, while a subset of motoneurons fire less reliably at higher frequencies (11 of 106 cells), the vast majority are recruited into the active pool in a way that matches their firing properties and dorsoventral position.

Next, we examined the voltage oscillations underlying the increases in firing rate and reliability during fictive swimming. To do this, voltage oscillations were grouped according to whether they were subthreshold or suprathreshold, both of which were then related to swimming frequency, as in Figure $7 J-L$. Once motoneurons began firing, the amplitude of oscillations remained constant over the full frequency range. However, for subthreshold oscillations, there was a consistent increase in amplitude related to swimming frequency. This observation sup- ports firing frequency measures, which suggested an increase in drive related to increases in swimming frequency. The only exception to this was found among the burst motoneurons, where the amplitude of subthreshold oscillations remained constant and close to suprathreshold levels over the full swimming frequency range (Fig. 7L). Again, this supports the observation that these motoneurons are more reliably activated at lower swimming frequencies.

Close examination of the values for suprathreshold oscillations in Figure 7J- $L$ reveals a systematic decrease in the average amplitude related to firing class. Thus, more intrinsically rhythmogenic, more excitable motoneurons may require less oscillatory drive to shape their firing activity appropriately. To see whether suprathreshold oscillations were systematically related to the current-evoked firing patterns and excitability of different motoneurons, average suprathreshold oscillation values were plotted against input resistance (Fig. $8 \mathrm{~A}$ ) and rheobase (Fig. $8 \mathrm{~B}$ ) according to firing class. Within the population as a whole, there was a clear correlation between the two measures. Oscillation amplitude was negatively correlated with input resistance $\left(R_{(104)}=-0.78 ; p<0.001 ; n=106\right)$ and positively correlated with rheobase $\left(R_{(104)}=0.78 ; p<0.001 ; n=106\right)$. More excitable cells had lower amplitude oscillations driving their firing activity. When related to firing class, tonic cells had the largest amplitude oscillations, and burst cells had the lowest amplitude oscillations, with chattering cells overlapping the two. The degree of overlap between the firing classes suggests that gradations in electrical properties are matched by gradations in oscillatory drive. To rule out that primary motoneurons were not biasing the dataset, we also performed correlations among the secondary population. Again, all of the statistically significant observations were maintained when we excluded primary motoneurons from the analysis (for statistical data, see Fig. 8 legend).

The fact that less excitable, tonic motoneurons exhibited larger amplitude oscillations during fictive swimming than more excitable, bursting motoneurons is consistent with the idea that tonic cells require more powerful, patterned synaptic drive to shape their rhythmic activity, than the more intrinsically bursting cells. This point is also obvious if the average peak firing frequency during swimming is used as some estimation of the amount of current required for motoneurons to fire. For example, during swimming, primaries fire at peak frequencies of $\sim 500$ $\mathrm{Hz}$ (Fig. 7E). For primaries to fire at that rate, current steps between 1.5 and $2 \times$ rheobase values are required (Fig. $5 H$ ). The same lookup table approach can also be used for secondary motoneurons $(\sim 400 \mathrm{~Hz}=1.5-2 \times$ rheobase $)$. Given the responses of different neurons to current steps in this range (Fig. $5 A-D$, right), it is clear that bursting cells could rely less on patterned drive to generate appropriate oscillatory firing responses, while tonic cells would have to rely on patterned drive more.

Are there also matching differences in the amount of depolarizing drive to get neurons to threshold? There is a difference in the reliability of firing as a function of firing class, from burst to chattering to tonic (Fig. 7I). A more relevant measure of recruitment would be the amount of depolarization from rest. When input resistance and rheobase were compared with the level of suprathreshold depolarization from rest at the on-cycle phase of swimming (Fig. 8C,D), on-cycle amplitude was negatively correlated with input resistance $\left(R_{(104)}=-0.73 ; p<0.001 ; n=106\right)$ and positively correlated with rheobase $\left(R_{(104)}=0.78 ; p<0.001\right.$; $n=106$ ). Thus, burst cells require less depolarizing drive to get them to fire, versus the tonic cells, with chattering cells again falling in between the two classes. 
A

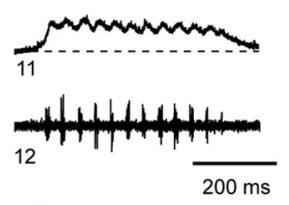

B
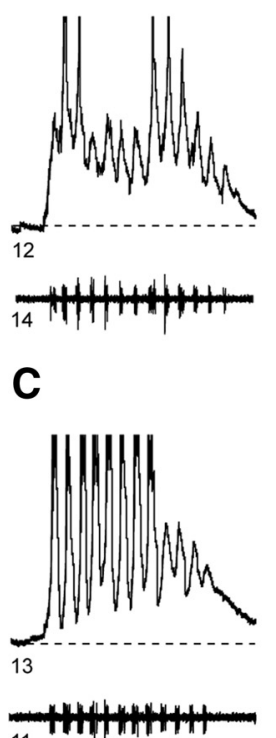

D

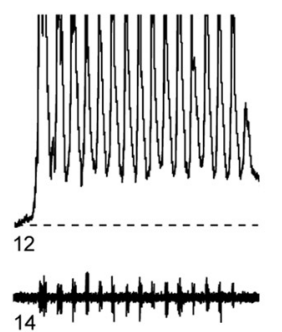

Swim episode
dRoP/Tonic

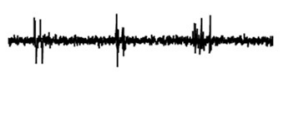

dS/Chattering
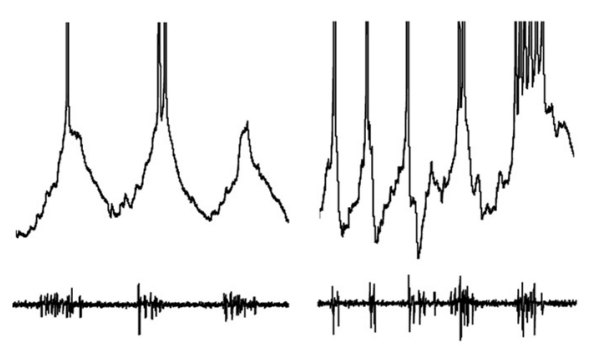

iS-c/Burst
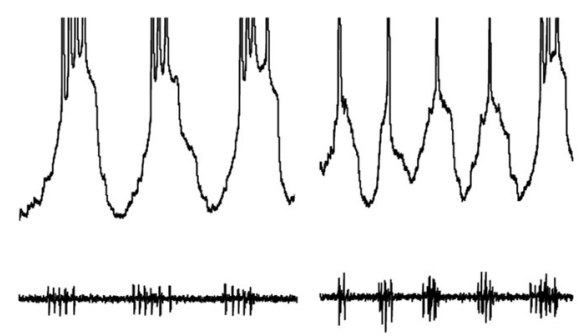

iS-nc/Burst

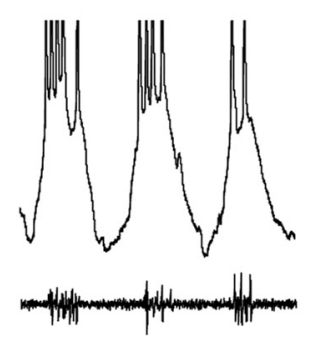

Low $\mathrm{Hz}$ swim

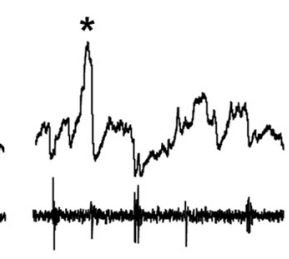

High Hz swim

\section{E}
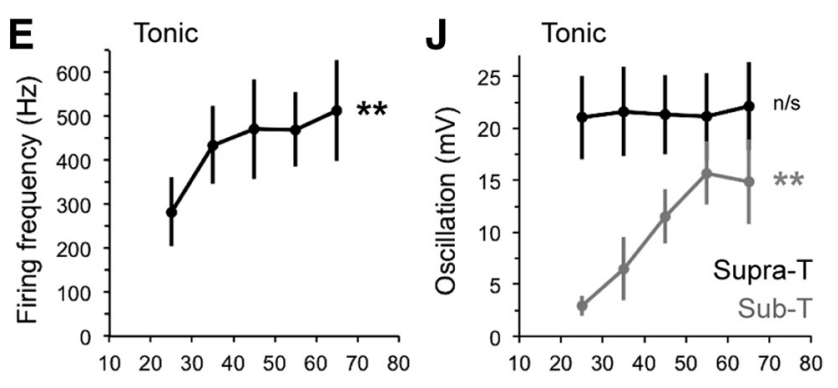

$\mathbf{F}$
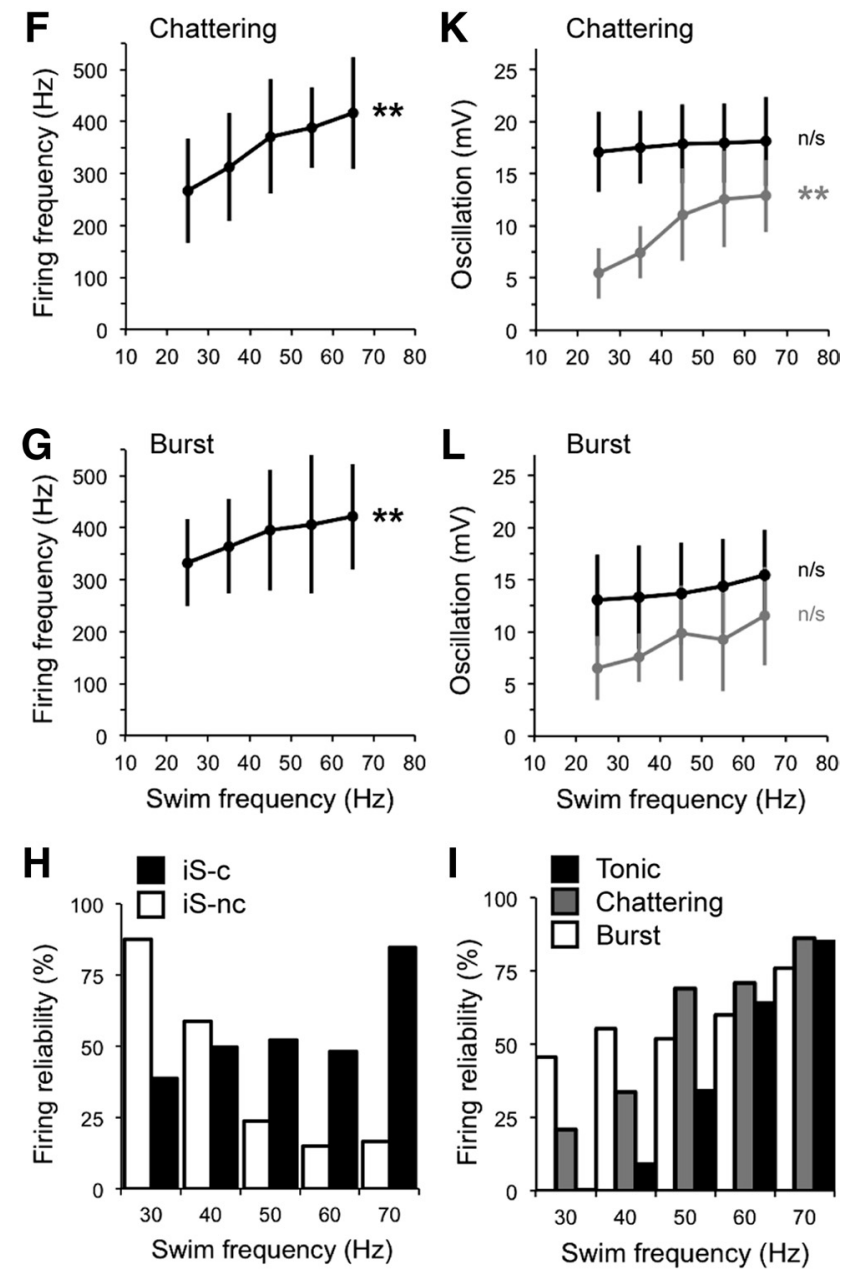

Figure 7. Differences in synaptic drive and the reliability of firing during fictive swimming related to anatomical class and firing type. $A-D$, Examples rhythmic oscillatory drive during fictive swimming in a dRoP tonic motoneuron $(\boldsymbol{A})$, a dS chattering motoneuron $(\boldsymbol{B})$, an iS-c bursting motoneuron (C), and an iS-nc bursting motoneuron (D). On the left is a whole episode at a slower time base. In the middle and right are examples of lower versus higher frequency (in hertz) swimming, respectively, at a faster time base. In $\boldsymbol{D}$, an asterisk marks a subthreshold oscillation, which is evident againsta barrage of synaptic activity. The segments from which simultaneous motoneuron (top) and peripheral motor nerve (bottom) recordings were performed are labeled below the respective traces. The dashed gray lines on the left indicate resting membrane potential. Calibration for all records is presented in $\boldsymbol{A}$. Note that the action potentials are truncated to more easily observe the oscillatory drive. $\boldsymbol{E}-\boldsymbol{G}$, Plots of firing frequency as a function of fictive swimming frequency from tonic $(\boldsymbol{E})$, chattering $(\boldsymbol{F})$, and burst $(\boldsymbol{G})$ motoneurons. Data points represent means \pm SDs plotted in $10 \mathrm{~Hz}$ bins. Tonic $(n=24)$, chattering $(n=45)$, burst $(n=20)$. Note that motoneurons were only included in this analysis if they fired two or more spikes over at least two swim frequency bin widths, so these represent a subset of the total population (89 of 106 cells). There is an increase in firing frequency as a function of swimming frequency for the different firing classes, consistent with their respective responses to increased current injection. The double black asterisks indicate significant differences using a pairwise comparison of firing frequency at the lowest and highest frequency of swimming tested on a cell-by-cell basis. Tonic: $421.3 \pm 19.5$ to $493 \pm 22.3 \mathrm{~Hz}\left(t_{(23)}=2.87 ; p<0.01 ; n=\right.$ 24). Chattering: $294.7 \pm 14.7$ to $362.2 \pm 17.5 \mathrm{~Hz}\left(t_{(44)}=4.93 ; p<0.001 ; n=45\right)$. Burst: $345.9 \pm 19.8$ to $398.0 \pm 29.2 \mathrm{~Hz}\left(t_{(19)}=2.69 ; p<0.05 ; n=20\right) . H$, Percentage of cycles in which a motoneuron fired an action potential (firing reliability) at a particular swim frequency for iS-c and is-nc motoneurons. For is-c motoneurons, this represents 2627 total cycles from 15 larvae between 20 and $70 \mathrm{~Hz}$, while for is-nc motoneurons it is 1427 total cycles from 11 larvae over the same frequency range. The iS-c class is unique in that it is de-recruited during increases in swimming frequency. I, The same reliability analysis as in $\boldsymbol{K}$, but organized according to firing type. Despite the iS-ncde-recruitment, there is an increase in firing reliability when considering the populations as defined by current-evoked firing pattern. Toniccells, 4793 cycles from 31 larvae; chattering cells, 8714 cycles from 53 larvae; burst cells, 3005 cycles from 22 larvae.J-L, Plots of suprathreshold (Supra-T) (black) and subthreshold (Sub-T) (gray) oscillations for tonic ( $)$ ), chattering $(\boldsymbol{K})$, and burst $(\boldsymbol{L})$ motoneurons. While tonic and chattering cells show a clear increase in subthreshold drive that eventually reaches suprathreshold levels at higher swim frequencies, burst neurons have subthreshold oscillations that do not increase significantly and are consistently closer to suprathreshold oscillations over the full swim frequency range. Data points represent the means \pm SDs plotted in 10 $\mathrm{Hz}$ bins. To the right of the data are indications of the significance of differences using a pairwise comparison of suprathreshold and subthreshold oscillations at the lowest and highest frequency of swimming tested on a cell-by-cell basis. * ${ }^{*}$ Significant; $n / s$, not significant. Tonic: subthreshold from $2.9 \pm 0.2$ to $14.2 \pm 0.9 \mathrm{mV}\left(t_{(30)}=11.85 ; p<0.001 ; n=31\right)$, suprathreshold from $21.4 \pm 0.7$ to $21.7 \pm 0.8 \mathrm{mV}$ $\left(t_{(30)}=0.62 ; p=0.538 ; n=31\right)$. Chattering: subthreshold, $5.6 \pm 0.3$ to $11.3 \pm 0.6 \mathrm{mV}\left(t_{(52)}=7.78 ; p<0.001 ; n=53\right)$; suprathreshold, $17.7 \pm 0.5$ to $18.0 \pm 0.6 \mathrm{mV}\left(t_{(52)}=0.74 ; p=0.462 ; n=53\right)$. Burst: subthreshold, $6.6 \pm 0.7$ to $9.1 \pm 1.0 \mathrm{mV}\left(t_{(21)}=1.98 ; p=0.063 ; n=22\right)$; suprathreshold, $13.6 \pm 1.1$ to $14.5 \pm 1.0 \mathrm{mV}\left(t_{(21)}=1.67 ; p=0.111 ; n=22\right)$. 

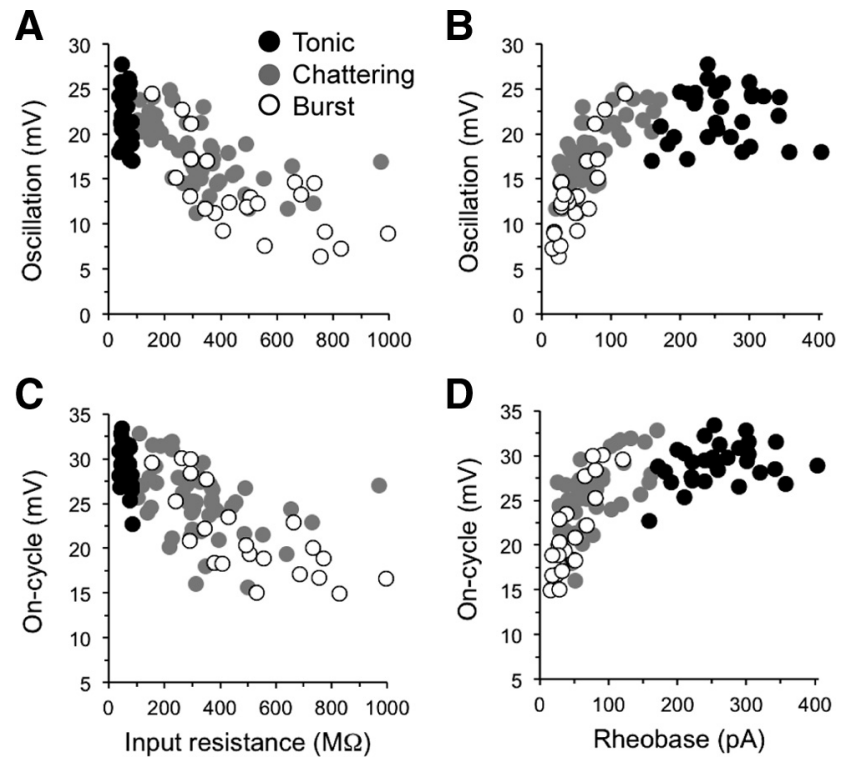

Figure 8. Oscillatory and depolarizing drive vary according to input resistance and rheobase. $\boldsymbol{A}$, Input resistance versus suprathreshold oscillation amplitude for each firing class. Input resistance is negatively correlated with the amplitude of suprathreshold oscillations for the entire population of motoneurons $\left(R_{(104)}=-0.78 ; p<0.001 ; n=106\right)$. Thus, higher input resistance motoneurons exhibit smaller amplitude membrane potential oscillations during swimming. The correlation is maintained when only the secondary motoneurons are examined $\left(R_{(73)}=-0.73 ; p<0.001 ; n=75\right) . \boldsymbol{B}$, Rheobase versus suprathreshold oscillation amplitude for each firing class. Rheobase is positively correlated with the amplitude of suprathreshold oscillations for the entire population of motoneurons $\left(R_{(104)}=0.78 ; p<0.001 ; n=106\right)$. Higher rheobase motoneurons exhibit larger-amplitude membrane potential oscillations during swimming. The correlation is maintained when only the secondary motoneurons are examined $\left(R_{(73)}=0.74 ; p<0.001 ; n=75\right)$. C, Input resistance versus suprathreshold on-cycle amplitude for each firing class. Input resistance is negatively correlated with on-cycle amplitude for the entire population of motoneurons $\left(R_{(104)}=-0.73 ; p<0.001 ; n=106\right)$. Higher input resistance motoneurons require less depolarizing drive to get them to fire. The correlation is maintained when only the secondary motoneurons are examined $\left(R_{(73)}=-0.63 ; p<0.001\right.$; $n=75)$. $\boldsymbol{D}$, Rheobase versus suprathreshold on-cycle amplitude for each firing class. Rheobase is positively correlated with on-cycle amplitude for the entire population of motoneurons $\left(R_{(104)}=0.78 ; p<0.001 ; n=106\right)$. Higher rheobase motoneurons require more depolarizing drive to get them to fire. The correlation is maintained when only the secondary motoneurons are examined $\left(R_{(73)}=0.74 ; p<0.001 ; n=75\right)$.

There are two features that could contribute to this observation, resting membrane potential and spike threshold. Resting membrane potential varied from -55.5 to $-75.7 \mathrm{mV}$, while threshold varied from -23 to $-48 \mathrm{mV}(n=106)$. When the two measures were compared, a significant negative correlation was found between resting membrane potential and on-cycle amplitude $\left(R_{(104)}=-0.46 ; p<0.001 ; n=106\right)$ and a significant positive correlation was found between spike threshold and oncycle amplitude $\left(R_{(104)}=0.30 ; p<0.01 ; n=106\right)$. These significant correlations were maintained when we examined only the secondary population (rest/on-cycle, $R_{(73)}=-0.45, p<0.001$, $n=75$; threshold/on-cycle, $\left.R_{(73)}=0.41, p<0.001, n=75\right)$. Therefore, the differences in suprathreshold on-cycle amplitude among the different classes reflect differences in the proximity of motoneurons to threshold; tonic cells have more hyperpolarized rests and more depolarized thresholds, while burst cells have more depolarized rests and more hyperpolarized thresholds. Chattering cells fall in between. These observations could help explain the order in which these neurons are recruited during swimming.

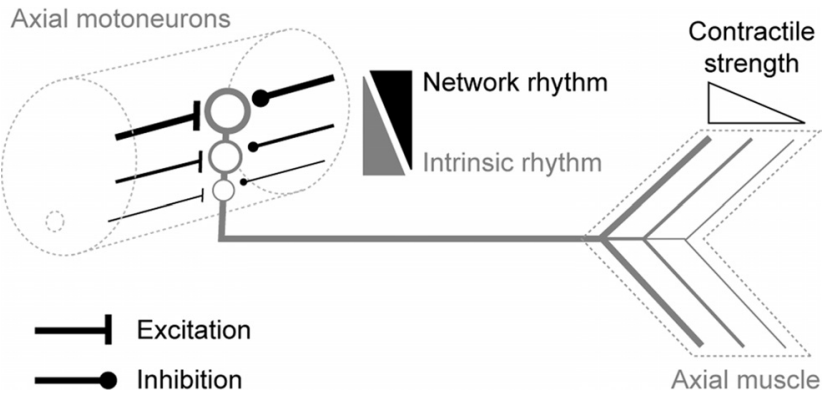

Figure 9. A model for the organization of the larval zebrafish axial locomotor network. Shown is a simple schematic that summarizes the implications of our findings. Intrinsic cellular properties like input resistance, rheobase, and current-evoked rhythmic bursting behavior are matched by gradients in excitatory and inhibitory synaptic drive. More excitable rhythmogenic neurons likely require less patterned drive to shape their rhythmic activity during swimming. During increases in swimming frequency, less rhythmogenic neurons, which rely more on patterned synaptic drive, are engaged. These systematic central differences in connectivity, intrinsic properties, and recruitment are translated peripherally into different intensities of local segmental muscle contractions, as observed during varying speeds of swimming.

\section{Discussion}

We have provided a systematic description of axial motoneuron anatomy, electrical properties, and synaptic drive during swimming, with the goal of matching these features to the topographic pattern of recruitment. In Figure 9, we present a model that summarizes our findings. Larger, less excitable neurons with little evidence of intrinsic rhythmicity likely require more highly patterned synaptic drive to activate and shape their oscillatory activity during swimming. In contrast, smaller, more excitable neurons with more intrinsic rhythmicity likely require less synaptic drive to activate and shape oscillatory activity, consistent with a larger role for endogenous rhythmicity in generating appropriately patterned motor output in these cells. These central patterns are translated peripherally to the appropriate skeletal muscle fibers. Although we have schematized this as three populations and grouped motoneurons into categories for the purposes of comparison, our data are actually more consistent with a continuum of anatomical, electrical, and synaptic properties.

The major implication from this work is that, within the spinal cord, there is more than one way to generate rhythmic locomotor output. This is something that has been long appreciated in the invertebrate motor control field (Marder and Bucher, 2007). We could reveal this pattern by identifying and recording from spinal neurons in an intact animal capable of its full range of locomotor movements. Many of our observations here confirm previous reports in other animals, in addition to humans. For example, we have shown that more forceful movements are produced not only by recruiting larger motoneurons/faster muscle fibers in accord with the well known size principle (Henneman, 1957; Mendell, 2005) but also by increasing the rate at which motoneurons fire once they are recruited (Monster and Chan, 1977). We have also demonstrated a closer proximity of smaller motoneurons to firing threshold, which would make them easier to recruit (Gabriel et al., 2003). The systematic tiling of the muscle segment by axial motoneurons of varying excitability is consistent with a recent study in Xenopus tadpoles (Zhang et al., 2011). Both Xenopus tadpoles and zebrafish larvae regulate their swimming speed through the water column by varying the frequency and amplitude of local body bends (von Seckendorff-Hoff and Wassersug, 1986; Müller and van Leeuwen, 2004; McLean et al., 2008; Green et al., 2011). Our findings now provide a clear anatomical substrate for the control of local bending intensity as well as for 
varying degrees of pitch and roll in zebrafish. If these shared observations are any indication, then differences in the contribution of endogenous versus network-based rhythmogenesis related to order of recruitment may also be a common feature of motor control systems.

\section{Predictions about zebrafish spinal network organization}

What is the source of rhythmic drive to motoneurons? In larval zebrafish, there are shifts in the activation of excitatory premotor interneurons during different frequencies of swimming. Interneurons that participate in slower movements are progressively inhibited, while interneurons that participate in faster movements are engaged (McLean et al., 2008). Prior work suggested a systematic addition of motoneurons to the active pool, but here we have observed a subset of motoneurons that drops out. This appears to be related to their selective innervation of superficial, slow skeletal muscle fibers and could explain why the drive to slow muscle fibers is reduced during higher frequencies of swimming (Buss and Drapeau, 2002). A similar observation has recently been made in older zebrafish, where some motoneurons drop out during more powerful escape movements (Kyriakatos et al., 2011). If slower premotor interneurons are the major source of excitatory drive to these motoneurons, then the fact that they drop out at higher frequencies could explain these observations. Alternatively, the same source of inhibition responsible for shutting down premotor interneurons could also be silencing these motoneurons.

Notwithstanding the silencing of a small subset of motoneurons, the vast majority of motoneurons fire more reliably with increases in swimming frequency. Since different premotor interneurons are responsible for the higher swimming frequencies, our observations lead to predictions about the nature of connectivity within the zebrafish spinal network. To influence more motoneurons, premotor neurons that drive faster, higherfrequency movements presumably make more extensive connections throughout the motor pool. The extent of premotor connectivity would then vary as a function of recruitment order, with premotor neurons activated at slower speeds connected to fewer motoneurons. Another possibility is that the motoneurons themselves are responsible for driving synchronous activity within the pool. The intraspinal collaterals are topographically organized, so more dorsal motoneurons could potentially drive cells located at their dorsoventral location and below. Premotor interneurons driving faster movements would only have to control the dorsal motoneurons, which would in turn regulate the activity of ventral motoneurons. The contribution of motoneurons to rhythmic drive during locomotion is not unprecedented (Perrins and Roberts, 1995; Mentis et al., 2005; Nishimaru et al., 2005). Based on our data, we also believe the weights of these connections are scaled appropriately according to the excitability of the motoneurons. Less excitable neurons receive more drive. This could be achieved in a number of ways. For instance, it could be that inputs to different motoneurons are similar in strength, but the degree of convergence varies as a function of excitability. Alternatively, individual connections could vary systematically, where a single premotor interneuron provides progressively more synaptic input to progressively less excitable motoneurons. In either case, a scaling of synaptic inputs would suggest that the recruitment order of motoneurons is not exclusively a function of their excitability (e.g., proximity to threshold) but also their activation by the appropriate premotor interneurons. Thus, the topographic pattern of recruitment in the larval zebrafish spinal cord is likely a combined function of cellular excitability and synaptic specificity.

By using an intact animal capable of its full range of locomotor frequencies, we have revealed topographic patterns of organization that relate morphological features to intrinsic excitability and synaptic drive. These data stand in contrast to a recent study in older zebrafish, which reported no relationship between measures of excitability and oscillatory drive, where excitatory input, if anything, shows a negative correlation with input resistance (Gabriel et al., 2011). However, those experiments were performed in a reduced preparation, where only slower frequencies of locomotion can be elicited, and the majority of drive to motoneurons is subthreshold. The resulting dataset of limited swimming frequencies may have caused those authors to miss the clear gradient in rhythmic oscillatory drive that we demonstrate here. Still, if we assume that the premotor interneurons driving the rhythmic activation of slower motoneurons are intact and functional in this preparation, then the synaptic scaling we propose may be manifested in connections between all premotor interneurons and their respective motoneuron targets. It will be important to identify the sources of presynaptic drive and the frequency at which interneurons and motoneurons are truly recruited, if we are to elucidate the principles governing spinal network connectivity and how they apply to the full range of movements.

\section{Broader implications}

In zebrafish, neural networks exhibit topographic patterns of functional organization due to their order of development (Kimura et al., 2006; McLean and Fetcho, 2009; Kinkhabwala et al., 2011; Koyama et al., 2011; Miri et al., 2011; Liao and Haehnel, 2012; Pujol-Martí et al., 2012; Satou et al., 2012). In the spinal cord, the networks that are recruited during the most powerful movements are the first to develop, with slower networks layered on as zebrafish grow into free-swimming larvae. The relative expression of different ion channels along the dorsoventral axis may also be a product of development, which could generate the heterogeneous firing patterns we have described here. Indeed, a relatively recent study has demonstrated differences in electrical properties of zebrafish motoneurons related to time of development (Moreno and Ribera, 2009). Thus, early-born networks may require more patterned, descending drive, while later-born ones require less. Previous work in Xenopus tadpoles suggests that reticulospinal neurons are an integral component of locomotor rhythm generating circuitry at embryonic stages (Soffe et al., 2009; Li et al., 2010), which is certainly consistent with this idea. Timing of development has also recently been implicated in the functional specification of limb motor networks (Tripodi et al., 2011; Benito-Gonzalez and Alvarez, 2012; Stam et al., 2012). Therefore, differences in endogenous oscillatory properties in the mammalian spinal cord could also reflect differences in recruitment order and time of development.

Since excitability and synaptic drive appear to be normalized in the zebrafish spinal cord, one potential implication from our work is that developmental programs can shape spinal network connectivity simply by regulating cellular excitability. Another implication is that later-born spinal networks are composed of diffusely connected, intrinsically rhythmogenic cells, while earlyborn networks represent a highly interconnected, less intrinsically rhythmogenic population. If so, then recruitment order may be a function of when motoneurons develop and the sequence in which different sources of presynaptic drive emerge. Future experiments examining the contribution of development to ana- 
tomical, physiological, and synaptic heterogeneity within the zebrafish spinal cord will help us test these predictions.

\section{References}

Asakawa K, Higashijima S, Kawakami K (2012) An mnr2b/hlxb9lb enhancer trap line that labels spinal and abducens motor neurons in zebrafish. Dev Dyn 241:327-332.

Balciunas D, Davidson AE, Sivasubbu S, Hermanson SB, Welle Z, Ekker SC (2004) Enhancer trapping in zebrafish using the Sleeping Beauty transposon. BMC Genomics 5:62.

Benito-Gonzalez A, Alvarez FJ (2012) Renshaw cells and Ia inhibitory interneurons are generated at different times from $\mathrm{p} 1$ progenitors and differentiate shortly after exiting the cell cycle. J Neurosci 32:1156-1170.

Brownstone RM, Wilson JM (2008) Strategies for delineating spinal locomotor rhythm-generating networks and the possible role of $\mathrm{Hb} 9$ interneurones in rhythmogenesis. Brain Res Rev 57:64-76.

Buss RR, Drapeau P (2000) Physiological properties of zebrafish embryonic red and white muscle fibers during early development. J Neurophysiol 84:1545-1557.

Buss RR, Drapeau P (2001) Synaptic drive to motoneurons during fictive swimming in the developing zebrafish. J Neurophysiol 86:197-210.

Buss RR, Drapeau P (2002) Activation of embryonic red and white muscle fibers during fictive swimming in the developing zebrafish. J Neurophysiol 87:1244-1251.

Buss RR, Bourque CW, Drapeau P (2003) Membrane properties related to the firing behavior of zebrafish motoneurons. J Neurophysiol 89:657-664.

del Bene F, Wyart C (2012) Optogenetics: a new enlightenment age for zebrafish neurobiology. Dev Neurobiol 72:404-414.

Drapeau P, Ali DW, Buss RR, Saint-Amant L (1999) In vivo recording from identifiable neurons of the locomotor network in the developing zebrafish. J Neurosci Methods 88:1-13.

Eisen JS, Pike SH, Romancier B (1990) An identified motoneuron with variable fates in embryonic zebrafish. J Neurosci 10:34-43.

Gabriel JP, Scharstein H, Schmidt J, Büschges A (2003) Control of flexor motoneuron activity during single leg walking of the stick insect on an electronically controlled treadwheel. J Neurobiol 56:237-251.

Gabriel JP, Ausborn J, Ampatzis K, Mahmood R, Eklöf-Ljunggren E, El Manira A (2011) Principles governing recruitment of motoneurons during swimming in zebrafish. Nat Neurosci 14:93-99.

Green MH, Ho RK, Hale ME (2011) Movement and function of the pectoral fins of the larval zebrafish (Danio rerio) during slow swimming. J Exp Biol 214:3111-3123.

Grillner S, Jessell TM (2009) Measured motion: searching for simplicity in spinal locomotor networks. Curr Opin Neurobiol 19:572-586.

Harris-Warrick RM (2010) General principles of rhythmogenesis in central pattern generator networks. Prog Brain Res 187:213-222.

Heitler WJ (2009) Practical tools for analysing rhythmic neural activity. J Neurosci Methods 185:151-164.

Henneman E (1957) Relation between size of neurons and their susceptibility to discharge. Science 126:1345-1347.

Higashijima S, Hotta Y, Okamoto H (2000) Visualization of cranial motor neurons in live transgenic zebrafish expressing green fluorescent protein under the control of the islet-1 promoter/enhancer. J Neurosci 20:206-218.

Higashijima S, Masino MA, Mandel G, Fetcho JR (2004) Engrailed-1 expression marks a primitive class of inhibitory spinal interneuron. J Neurosci 24:5827-5839.

Husch A, Cramer N, Harris-Warrick RM (2011) Long-duration perforated patch recordings from spinal interneurons of adult mice. J Neurophysiol 106:2783-2789.

Kiehn O, Kjaerulff O, Tresch MC, Harris-Warrick RM (2000) Contributions of intrinsic motor neuron properties to the production of rhythmic motor output in the mammalian spinal cord. Brain Res Bull 53:649-659.

Kimura Y, Okamura Y, Higashijima S (2006) alx, a zebrafish homolog of Chx10, marks ipsilateral descending excitatory interneurons that participate in the regulation of spinal locomotor circuits. J Neurosci 26:5684-5697.

Kinkhabwala A, Riley M, Koyama M, Monen J, Satou C, Kimura Y, Higashijima S, Fetcho J (2011) A structural and functional ground plan for neurons in the hindbrain of zebrafish. Proc Natl Acad Sci U S A 108:1164-1169.

Koyama M, Kinkhabwala A, Satou C, Higashijima S, Fetcho J (2011) Map- ping a sensory-motor network onto a structural and functional ground plan in the hindbrain. Proc Natl Acad Sci U S A 108:1170-1175.

Kyriakatos A, Mahmood R, Ausborn J, Porres CP, Büschges A, El Manira A (2011) Initiation of locomotion in adult zebrafish. J Neurosci 31:8422-8431.

Liao JC, Haehnel M (2012) Physiology of afferent neurons in larval zebrafish provides a functional framework for lateral line somatotopy. J Neurophysiol 107:2615-2623.

Li WC (2011) Generation of locomotion rhythms without inhibition in vertebrates: the search for pacemaker neurons. Integr Comp Biol 51: $879-889$

Li WC, Roberts A, Soffe SR (2010) Specific brainstem neurons switch each other into pacemaker mode to drive movement by activating NMDA receptors. J Neurosci 30:16609-16620.

Luna VM, Wang M, Ono F, Gleason MR, Dallman JE, Mandel G, Brehm P (2004) Persistent electrical coupling and locomotory dysfunction in the zebrafish mutant shocked. J Neurophysiol 92:2003-2009.

Marder E, Bucher D (2007) Understanding circuit dynamics using the stomatogastric nervous system of lobsters and crabs. Annu Rev Physiol 69:291-316.

Masino MA, Fetcho JR (2005) Fictive swimming motor patterns in wild type and mutant larval zebrafish. J Neurophysiol 93:3177-3188.

McLean DL, Fetcho JR (2009) Spinal interneurons differentiate sequentially from those driving the fastest swimming movements in larval zebrafish to those driving the slowest ones. J Neurosci 29:13566-13577.

McLean DL, Fetcho JR (2011) Movement, technology and discovery in the zebrafish. Curr Opin Neurobiol 21:110-115.

McLean DL, Fan J, Higashijima S, Hale ME, Fetcho JR (2007) A topographic map of recruitment in spinal cord. Nature 446:71-75.

McLean DL, Masino MA, Koh IY, Lindquist WB, Fetcho JR (2008) Continuous shifts in the active set of spinal interneurons during changes in locomotor speed. Nat Neurosci 11:1419-1429.

Mendell LM (2005) The size principle: a rule describing the recruitment of motoneurons. J Neurophysiol 93:3024-3026.

Menelaou E, Svoboda KR (2009) Secondary motoneurons in juvenile and adult zebrafish: axonal pathfinding errors caused by embryonic nicotine exposure. J Comp Neurol 512:305-322.

Mentis GZ, Alvarez FJ, Bonnot A, Richards DS, Gonzalez-Forero D, Zerda R, O'Donovan MJ (2005) Noncholinergic excitatory actions of motoneurons in the neonatal mammalian spinal cord. Proc Natl Acad Sci U S A 102:7344-7349.

Miri A, Daie K, Arrenberg AB, Baier H, Aksay E, Tank DW (2011) Spatial gradients and multidimensional dynamics in a neural integrator circuit. Nat Neurosci 14:1150-1159.

Monster AW, Chan H (1977) Isometric force production by motor units of extensor digitorum communis muscle in man. J Neurophysiol 40:1432-1443.

Moreno RL, Ribera AB (2009) Zebrafish motor neuron subtypes differ electrically prior to axonal outgrowth. J Neurophysiol 102:2477-2484.

Moreno RL, Ribera AB (2010) Developmental regulation of subtypespecific motor neuron excitability. Ann N Y Acad Sci 1198:201-207.

Müller UK, van Leeuwen JL (2004) Swimming of larval zebrafish: ontogeny of body waves and implications for locomotory development. J Exp Biol 207:853-868.

Myers PZ (1985) Spinal motoneurons of the larval zebrafish. J Comp Neurol 236:555-561.

Myers PZ, Eisen JS, Westerfield M (1986) Development and axonal outgrowth of identified motoneurons in the zebrafish. J Neurosci 6:22782289.

Nishimaru H, Restrepo CE, Ryge J, Yanagawa Y, Kiehn O (2005) Mammalian motor neurons corelease glutamate and acetylcholine at central synapses. Proc Natl Acad Sci U S A 102:5245-5249.

Perrins R, Roberts A (1995) Cholinergic and electrical synapses between synergistic spinal motoneurones in the Xenopus laevis embryo. J Physiol 485:135-144.

Pujol-Martí J, Zecca A, Baudoin JP, Faucherre A, Asakawa K, Kawakami K, López-Schier H (2012) Neuronal birth order identifies a dimorphic sensorineural map. J Neurosci 32:2976-2987.

Robinson DW, Cameron WE (2000) Time-dependent changes in input resistance of rat hypoglossal motoneurons associated with whole-cell recording. J Neurophysiol 83:3160-3164. 
Saint-Amant L (2010) Development of motor rhythms in zebrafish embryos. Prog Brain Res 187:47-61.

Satou C, Kimura Y, Higashijima S (2012) Generation of multiple classes of V0 neurons in zebrafish spinal cord: progenitor heterogeneity and temporal control of neuronal diversity. J Neurosci 32:1771-1783.

Schmidt BJ, Hochman S, MacLean JN (1998) NMDA receptor-mediated oscillatory properties: potential role in rhythm generation in the mammalian spinal cord. Ann N Y Acad Sci 860:189-202.

Sillar KT, Reith CA, McDearmid JR (1998) Development and aminergic neuromodulation of a spinal locomotor network controlling swimming in Xenopus larvae. Ann N Y Acad Sci 860:318-332.

Soffe SR, Roberts A, Li WC (2009) Defining the excitatory neurons that drive the locomotor rhythm in a simple vertebrate: insights into the origin of reticulospinal control. J Physiol 587:4829-4844.

Stam FJ, Hendricks TJ, Zhang J, Geiman EJ, Francius C, Labosky PA, Clotman F, Goulding M (2012) Renshaw cell interneuron specialization is controlled by a temporally restricted transcription factor program. Development 139:179-190.

Tripodi M, Stepien AE, Arber S (2011) Motor antagonism exposed by spatial segregation and timing of neurogenesis. Nature 479:61-66.

Uemura O, Okada Y, Ando H, Guedj M, Higashijima S, Shimazaki T, Chino $\mathrm{N}$, Okano H, Okamoto H (2005) Comparative functional genomics re- vealed conservation and diversification of three enhancers of the isll gene for motor and sensory neuron-specific expression. Dev Biol 278:587-606.

van Raamsdonk W, Pool CW, te Kronnie G (1978) Differentiation of muscle fiber types in the teleost Brachydanio rerio. Anat Embryol (Berl) 153:137-155.

van Raamsdonk W, van't Veer L, Veeken K, Heyting C, Pool CW (1982) Differentiation of muscle fiber types in the teleost Brachydanio rerio, the zebrafish. Posthatching development. Anat Embryol (Berl) 164:51-62.

von Seckendorff-Hoff K, Wassersug RJ (1986) The kinematics of swimming in larvae of the clawed frog, Xenopus laevis. J Exp Biol 122:1-12.

Wen H, Brehm P (2005) Paired motor neuron-muscle recordings in zebrafish test the receptor blockade model for shaping synaptic current. J Neurosci 25:8104-8111.

Westerfield M, McMurray JV, Eisen JS (1986) Identified motoneurons and their innervation of axial muscles in the zebrafish. J Neurosci 6:2267-2277.

Zhang HY, Issberner J, Sillar KT (2011) Development of a spinal locomotor rheostat. Proc Natl Acad Sci U S A 108:11674-11679.

Ziskind-Conhaim L, Mentis GZ, Wiesner EP, Titus DJ (2010) Synaptic integration of rhythmogenic neurons in the locomotor circuitry: the case of Hb9 interneurons. Ann N Y Acad Sci 1198:72-84. 